\title{
Gradient-Based Electrical Conductivity Imaging Using MR Phase
}

\author{
Necip Gurler and Yusuf Ziya Ider*
}

Purpose: To develop a fast, practically applicable, and boundary artifact free electrical conductivity imaging method that does not use transceive phase assumption, and that is more robust against the noise.

Theory: Starting from the Maxwell's equations, a new electrical conductivity imaging method that is based solely on the MR transceive phase has been proposed. Different from the previous phase based electrical properties tomography (EPT) method, a new formulation was derived by including the gradients of the conductivity into the equations.

Methods: The governing partial differential equation, which is in the form of a convection-reaction-diffusion equation, was solved using a three-dimensional finite-difference scheme. To evaluate the performance of the proposed method numerical simulations, phantom and in vivo human experiments have been conducted at 3T.

Results: Simulation and experimental results of the proposed method and the conventional phase-based EPT method were illustrated to show the superiority of the proposed method over the conventional method, especially in the transition regions and under noisy data.

Conclusion: With the contributions of the proposed method to the phase-based EPT approach, a fast and reliable electrical conductivity imaging appears to be feasible, which is promising for clinical diagnoses and local SAR estimation. Magn Reson Med 77:137-150, 2017. ( 2016 Wiley Periodicals, Inc.

Key words: MREPT; phase based; conductivity; boundary artifact

\section{INTRODUCTION}

Imaging electrical properties (EPs, conductivity $(\sigma)$ and permittivity $(\epsilon)$ ) of tissues can potentially be useful in several applications. For example, conductivity is a key parameter in the calculation of the specific absorption rate (SAR) map of a patient, which is a crucial issue at high field MRI. Additionally, EPs can be used for diagnostic purposes. In in vivo studies, especially in oncology, it has been shown that the conductivity of a tumor region increases (1-3). Furthermore, EPs may also be used in therapy monitoring (or planning) such as transcranial magnetic stimulation (TMS) (4), hyperthermia treatment (5), and radiofrequency (RF) ablation (6).

Department of Electrical and Electronics Engineering, Bilkent University, Ankara, Turkey

Grant sponsor: TUBITAK; Grant number: 114E522.

*Correspondence to: Yusuf Ziya Ider, PhD, Bilkent University, Ankara, Turkey. E-mail: ider@ee.bilkent.edu.tr

Received 25 August 2015; revised 27 November 2015; accepted 27 November 2015

DOI 10.1002/mrm.26097

Published online 13 January 2016 in Wiley Online Library (wileyonlinelibrary. com).

(C) 2016 Wiley Periodicals, Inc.
Over the years, many methods have been proposed to image EPs at various frequencies. For low-frequency applications (1 kHz to $1 \mathrm{MHz}$ ), electrical impedance tomography (EIT) and magnetic induction tomography (MIT) have been developed to calculate EPs (7-12). In these methods, sinusoidal currents are either injected into tissue through surface electrodes (EIT) or induced in the tissue using external coils (MIT), and induced voltages are measured between surface electrodes. The current-voltage data sets are used to calculate impedance maps, but the resulting images lack spatial resolution because of the insensitivities of the surface measurements to inner regions. To improve the spatial resolution, magnetic resonance electrical impedance tomography (MREIT) has been proposed (13-20). In MREIT, additional magnetic field is generated by injecting currents into the tissue through surface electrodes, and this additional magnetic field is then measured using an MRI scanner to reconstruct EPs. Because the permittivity effect can be ignored for the frequencies below $10 \mathrm{kHz}$, these studies generally focus on imaging the conductivity.

Recently, a method that is used to image EPs at Larmor frequency, called magnetic resonance electrical properties tomography (MREPT), has been proposed by Katscher et al (21). It was first introduced by Haacke et al (22), and it was practically applied by Wen et al (23). The idea is elegant in its simplicity, and it is based on the calculation of EPs from the perturbed RF magnetic field of an MRI system, resulting from the presence of the object. The relation between the magnetic flux density, $\mathbf{B}=\left(\mathrm{B}_{\mathrm{x}}, \mathrm{B}_{\mathrm{y}}, \mathrm{B}_{\mathrm{z}}\right)$, and the complex permittivity of an object to be imaged in MRI, $\gamma=\sigma+i \omega \epsilon$, can be described using the $\mathrm{e}^{\mathrm{i} \omega \mathrm{t}}$ convention as follows:

$$
-\nabla^{2} \mathbf{B}=\frac{\nabla \gamma}{\gamma} \times(\nabla \times \mathbf{B})-i \omega \mu_{0} \gamma \mathbf{B}
$$

where $\mathbf{B}$ and $\gamma$ are functions of space, $\mathbf{r}=(x, y, z), \omega$ is the Larmor frequency, and $\mu_{0}$ is the free space permeability.

If one assumes that EPs are locally constant in the tissue compartments, the gradient term $\left(\frac{\nabla \gamma}{\gamma} \times(\nabla \times \mathbf{B})\right)$ in Eq. [1] vanishes. Rewriting the rest in terms of transmit sensitivity $\left(B_{1}^{+}\right)$and receive sensitivity $\left(B_{1}^{-}\right)$(24), EPs can be found as

$$
\gamma=\frac{\nabla^{2} \mathrm{~B}_{1}^{ \pm}}{\mathrm{i} \omega \mu_{0} \mathrm{~B}_{1}^{ \pm}}
$$

Eq. [2] is the central equation of many EPT studies, but the use of this equation has several drawbacks and challenges. One such challenge is the well-known "boundary artifact" issue. Because $\nabla \gamma$ is assumed to be zero, methods that are based on Eq. [2] are not capable of reconstructing EPs in the 
transition regions (or boundaries) of tissues. For complex structures, this problem can easily lead to misinterpretation of EP images. To tackle the boundary artifact issue, several approaches have been proposed so far (25-27). In (25), a convection-reaction partial differential equation (PDE) based formulation (cr-MREPT) was introduced, in which the PDE is solved using the finite-element method. In (26), a gradient-based EPT (gEPT) algorithm was proposed, which has the same formulation as in (25), but uses multichannel transceiver RF coil to provide multiple transmit and receive fields for solving the PDE. In (27), an iterative approach based on the integral equation of electromagnetic field is proposed.

The other issue is the transceive phase assumption (TPA). To apply Eq. [2], one needs to have both magnitude and phase information of transmit (or receive) sensitivity. $\mathrm{B}_{1}^{+}$magnitude can be measured using any $\mathrm{B}_{1}$-mapping technique (28-31). However, $\mathrm{B}_{1}^{+}$phase cannot be measured directly in MRI. A temporary solution for this problem is to make a rough approximation for birdcage-like quadrature coil configurations in which the transmit phase is approximated as half of the transceive phase $(21,23)$. To solve this issue completely, multichannel transceiver configuration-based studies have been proposed (32-35). In (32), local Maxwell tomography (LMT), a TPA-free formulation, was derived and EPs were solved analytically using transmit and receive sensitivity distributions of multiple coils. However, because LMT uses Eq. [2], it is still faced with the boundary issue. Its generalized version (33), which takes varying EPs and magnetization into consideration, has also been proposed, but still needs to be assessed in practice. In (34), absolute RF phase was estimated using a large-scale optimization algorithm by making elliptical symmetry assumption, and EPs were calculated again based on Eq. [2]. In (35), a novel singleacquisition EPT based on the relative receive coil sensitivities was proposed. The formulation is based on Eq. [2], and third-order derivatives are necessary to calculate EPs, which makes the method more sensitive to noisy data. Apart from multichannel configuration studies, a more practical method, called the phase-based EPT (36-38), can be used to eliminate transceive phase approximation. This method calculates only the conductivity using MR transceive phase and therefore does not require $B_{1}$-mapping. Hence, it is considerably fast when compared with $\mathrm{B}_{1^{-}}$mapping-based EPT methods. However, in its current form, the boundary artifact issue precludes the clinical applications of this method.

Last but not least is the signal-to-noise (SNR) issue. Because most of the EPT methods use the Laplacian of the RF magnetic field, they are all sensitive to noise. Therefore, it is extremely important to obtain high SNR MR images to get high-quality EP maps. Quantitative analysis of SNR in MREPT is well documented in a recent paper (39).

By considering these drawbacks and challenges, the missing piece in this puzzle may be a fast, practically applicable, and boundary artifact-free MREPT method that does not use transceive phase assumption. To reach this goal, in this study, a new formulation for the phasebased EPT method was made by including the EP gradient terms. A partial differential equation is derived in the form of a convection-reaction equation. Different from the previous studies $(25,40)$, a finite-difference formulation was used on a Cartesian grid. The feasibility of this approach is demonstrated in simulations, phantom experiments, and in vivo human experiments.

\section{THEORY}

A convection reaction equation-based MREPT formula $(25,40)$ can be written in its logarithm form as follows (the complete derivation is found in Appendix A):

$$
\boldsymbol{\beta}^{ \pm} \cdot \nabla \ln (\gamma)-\nabla^{2} \mathrm{~B}_{1}^{ \pm}+\mathrm{i} \omega \mu_{0} \gamma \mathrm{B}_{1}^{ \pm}=0
$$

where

$$
\begin{aligned}
\gamma=\sigma+i \omega \epsilon, \quad \nabla \ln (\gamma)=\left[\begin{array}{c}
\frac{\partial \ln (\gamma)}{\partial \mathrm{x}} \\
\frac{\partial \ln (\gamma)}{\partial \mathrm{y}} \\
\frac{\partial \ln (\gamma)}{\partial \mathrm{z}}
\end{array}\right], \quad \boldsymbol{\beta}^{ \pm}=\left[\begin{array}{c}
\beta_{\mathrm{x}}^{ \pm} \\
\beta_{\mathrm{y}}^{ \pm} \\
\beta_{\mathrm{z}}^{ \pm}
\end{array}\right] \\
=\left[\begin{array}{c}
\frac{\partial \mathrm{B}_{1}^{ \pm}}{\partial \mathrm{x}} \mp \mathrm{i} \frac{\partial \mathrm{B}_{1}^{ \pm}}{\partial \mathrm{y}}+\frac{1}{2} \frac{\partial \mathrm{B}_{\mathrm{z}}}{\partial \mathrm{z}} \\
\pm \mathrm{i} \frac{\partial \mathrm{B}_{1}^{ \pm}}{\partial \mathrm{x}}+\frac{\partial \mathrm{B}_{1}^{ \pm}}{\partial \mathrm{y}} \pm \mathrm{i} \frac{1}{2} \frac{\partial \mathrm{B}_{\mathrm{z}}}{\partial \mathrm{z}} \\
-\frac{1}{2} \frac{\partial \mathrm{B}_{\mathrm{z}}}{\partial \mathrm{x}} \mp \mathrm{i} \frac{1}{2} \frac{\partial \mathrm{B}_{\mathrm{z}}}{\partial \mathrm{y}}+\frac{\partial \mathrm{B}_{1}^{ \pm}}{\partial \mathrm{z}}
\end{array}\right]
\end{aligned}
$$

To derive the phase-based formula, each term in Eq. [3] is written in terms of magnitude and phase. Substituting $\mathrm{B}_{1}^{ \pm}=\left|\mathrm{B}_{1}^{ \pm}\right| \mathrm{e}^{\mathrm{i} \varphi^{ \pm}}$where $\varphi^{+}$and $\varphi^{-}$are transmit and receive phases, respectively, and assuming $\nabla\left|\mathrm{B}_{1}^{+}\right|=0$ and $\nabla\left|\mathrm{B}_{1}^{-}\right|=0$, yields

$$
\begin{aligned}
& \beta_{\mathrm{x}}^{+}=\left|\mathrm{B}_{1}^{+}\right| \mathrm{e}^{\mathrm{i} \varphi^{+}}\left(\mathrm{i} \frac{\partial}{\partial \mathrm{x}} \varphi^{+}+\frac{\partial}{\partial \mathrm{y}} \varphi^{+}\right)+\frac{1}{2} \frac{\partial \mathrm{B}_{\mathrm{z}}}{\partial \mathrm{z}} \\
& \beta_{\mathrm{y}}^{+}=\left|\mathrm{B}_{1}^{+}\right| \mathrm{e}^{\mathrm{i} \varphi^{+}}\left(-\frac{\partial}{\partial \mathrm{x}} \varphi^{+}+\mathrm{i} \frac{\partial}{\partial \mathrm{y}} \varphi^{+}\right)+\mathrm{i} \frac{1}{2} \frac{\partial \mathrm{B}_{\mathrm{z}}}{\partial \mathrm{z}} \\
& \beta_{\mathrm{z}}^{+}=\left|\mathrm{B}_{1}^{+}\right| \mathrm{e}^{\mathrm{i} \varphi^{+}}\left(\mathrm{i} \frac{\partial}{\partial \mathrm{z}} \varphi^{+}\right)-\frac{1}{2} \frac{\partial \mathrm{B}_{\mathrm{z}}}{\partial \mathrm{x}}-\mathrm{i} \frac{1}{2} \frac{\partial \mathrm{B}_{\mathrm{z}}}{\partial \mathrm{y}} \\
& \nabla^{2} \mathrm{~B}_{1}^{+}=\left|\mathrm{B}_{1}^{+}\right| \mathrm{e}^{\mathrm{i} \varphi^{+}}\left(-\left(\frac{\partial}{\partial \mathrm{x}} \varphi^{+} \frac{\partial}{\partial \mathrm{x}} \varphi^{+}+\frac{\partial}{\partial \mathrm{y}} \varphi^{+} \frac{\partial}{\partial \mathrm{y}} \varphi^{+}\right.\right. \\
& \left.\left.+\frac{\partial}{\partial \mathrm{z}} \varphi^{+} \frac{\partial}{\partial \mathrm{z}} \varphi^{+}\right)+\mathrm{i} \nabla^{2} \varphi^{+}\right) \\
& \beta_{\mathrm{x}}^{-}=\left|\mathrm{B}_{1}^{-}\right| \mathrm{e}^{\mathrm{i} \varphi^{-}}\left(\mathrm{i} \frac{\partial}{\partial \mathrm{x}} \varphi^{-}-\frac{\partial}{\partial \mathrm{y}} \varphi^{-}\right)+\frac{1}{2} \frac{\partial \mathrm{B}_{\mathrm{z}}}{\partial \mathrm{z}} \\
& \beta_{\mathrm{y}}^{-}=\left|\mathrm{B}_{1}^{-}\right| \mathrm{e}^{\mathrm{i} \varphi^{-}}\left(\frac{\partial}{\partial \mathrm{x}} \varphi^{-}+\mathrm{i} \frac{\partial}{\partial \mathrm{y}} \varphi^{-}\right)-\mathrm{i} \frac{1}{2} \frac{\partial \mathrm{B}_{\mathrm{z}}}{\partial \mathrm{z}} \\
& \beta_{\mathrm{z}}^{-}=\left|\mathrm{B}_{1}^{-}\right| \mathrm{e}^{\mathrm{i} \varphi^{-}}\left(\mathrm{i} \frac{\partial}{\partial \mathrm{z}} \varphi^{-}\right)-\frac{1}{2} \frac{\partial \mathrm{B}_{\mathrm{z}}}{\partial \mathrm{x}}+\mathrm{i} \frac{1}{2} \frac{\partial \mathrm{B}_{\mathrm{z}}}{\partial \mathrm{y}} \\
& \nabla^{2} \mathrm{~B}_{1}^{-}=\left|\mathrm{B}_{1}^{-}\right| \mathrm{e}^{\mathrm{i} \varphi^{-}}\left(-\left(\frac{\partial}{\partial \mathrm{x}} \varphi^{-} \frac{\partial}{\partial \mathrm{x}} \varphi^{-}+\frac{\partial}{\partial \mathrm{y}} \varphi^{-} \frac{\partial}{\partial \mathrm{y}} \varphi^{-}\right.\right. \\
& \left.\left.+\frac{\partial}{\partial \mathrm{z}} \varphi^{-} \frac{\partial}{\partial \mathrm{z}} \varphi^{-}\right)+\mathrm{i} \nabla^{2} \varphi^{-}\right)
\end{aligned}
$$


Substituting Eqs. [4-11] into Eq. [3], common terms $\left(\left|\mathrm{B}_{1}^{ \pm}\right| \mathrm{e}^{\mathrm{i} \varphi^{ \pm}}\right)$will cancel and yield the following transmit or receive phase-based EPT formulas:

$$
\begin{aligned}
\Omega^{ \pm} \cdot \nabla \ln (\gamma) & +\left(\left(\left(\frac{\partial}{\partial \mathrm{x}} \varphi^{ \pm}\right)^{2}+\left(\frac{\partial}{\partial \mathrm{y}} \varphi^{ \pm}\right)^{2}\right.\right. \\
& \left.\left.+\left(\frac{\partial}{\partial \mathrm{z}} \varphi^{ \pm}\right)^{2}\right)-\mathrm{i} \nabla^{2} \varphi^{ \pm}\right)+i \omega \mu_{0} \gamma=0
\end{aligned}
$$

where

$$
\begin{aligned}
\Omega^{ \pm}= & {\left[\begin{array}{c}
\Omega_{\mathrm{x}}^{ \pm} \\
\Omega_{\mathrm{y}}^{ \pm} \\
\Omega_{\mathrm{z}}^{ \pm}
\end{array}\right] } \\
= & {\left[\begin{array}{c}
\mathrm{i} \frac{\partial}{\partial \mathrm{x}} \varphi^{ \pm} \pm \frac{\partial}{\partial \mathrm{y}} \varphi^{ \pm} \\
\mp \frac{\partial}{\partial \mathrm{x}} \varphi^{ \pm}+\mathrm{i} \frac{\partial}{\partial \mathrm{y}} \varphi^{ \pm} \\
\mathrm{i} \frac{\partial}{\partial \mathrm{z}} \varphi^{ \pm}
\end{array}\right]+\frac{1}{\mathrm{~B}_{1}^{ \pm}}\left[\begin{array}{c}
\frac{1}{2} \frac{\partial \mathrm{B}_{\mathrm{z}}}{\partial \mathrm{z}} \\
\pm \mathrm{i} \frac{1}{2} \frac{\partial \mathrm{B}_{\mathrm{z}}}{\partial \mathrm{z}} \\
-\frac{1}{2} \frac{\partial \mathrm{B}_{\mathrm{z}}}{\partial \mathrm{x}} \mp \mathrm{i} \frac{1}{2} \frac{\partial \mathrm{B}_{\mathrm{z}}}{\partial \mathrm{y}}
\end{array}\right] }
\end{aligned}
$$

There are several issues with using Eq. [12]. First, $B_{z}$ cannot be measured using MRI, and it has to be estimated. Alternatively, we can make the assumption that the first derivatives of $B_{z}$ with respect to each spatial component ( $\mathrm{x}, \mathrm{y}$, and $\mathrm{z}$ ) are negligible when compared with $\mathrm{B}_{1}^{+}$and $\mathrm{B}_{1}^{-}$in the region of interest. By doing so, the second term of $\Omega^{ \pm}$will be very small when compared with the first term of $\Omega^{ \pm}$, and therefore this term may be neglected. Second, the transmit or receive phase must be known so that EPs can be calculated using this equation. However, we can only measure the transceive phase using $\operatorname{MRI}\left(\varphi^{\operatorname{tr}}=\varphi^{+}+\varphi^{-}\right)$; therefore, we need to go one step further and write the equation in terms of $\varphi^{\text {tr }}$. To do this, we sum the transmit and receive phase-based EPT equations (Eq. [12]) and obtain

$$
\left[\begin{array}{c}
\left(\mathrm{i} \frac{\partial}{\partial \mathrm{x}} \varphi^{\mathbf{t r}}+\frac{\partial}{\partial \mathrm{y}}\left(\varphi^{+}-\varphi^{-}\right)\right) \\
\left(\frac{\partial}{\partial \mathrm{x}}\left(-\varphi^{+}+\varphi^{-}\right)+\mathrm{i} \frac{\partial}{\partial \mathrm{y}} \varphi^{\mathbf{t r}}\right) \\
i \frac{\partial}{\partial \mathrm{z}} \varphi^{\operatorname{tr}}
\end{array}\right] \cdot\left[\begin{array}{c}
\frac{\partial \ln (\gamma)}{\partial \mathrm{x}} \\
\frac{\partial \ln (\gamma)}{\partial \mathrm{y}} \\
\frac{\partial \ln (\gamma)}{\partial \mathrm{z}}
\end{array}\right]
$$

where

$$
\begin{aligned}
\mathrm{k}_{\text {real }} & =\left(\frac{\partial}{\partial \mathrm{x}} \varphi^{\mathrm{tr}}\right)^{2}+\left(\frac{\partial}{\partial \mathrm{y}} \varphi^{\mathrm{tr}}\right)^{2}+\left(\frac{\partial}{\partial \mathrm{z}} \varphi^{\mathrm{tr}}\right)^{2} \\
& -2\left(\frac{\partial}{\partial \mathrm{x}} \varphi^{-} \frac{\partial}{\partial \mathrm{x}} \varphi^{+}+\frac{\partial}{\partial \mathrm{y}} \varphi^{-} \frac{\partial}{\partial \mathrm{y}} \varphi^{+}+\frac{\partial}{\partial \mathrm{z}} \varphi^{-} \frac{\partial}{\partial \mathrm{z}} \varphi^{+}\right)
\end{aligned}
$$

In Eq. [13], conductivity $(\sigma)$ is related with the imaginary terms. Writing only imaginary terms and assuming that $\sigma^{2} \gg(\omega \epsilon)^{2}$ yields our central equation (the complete derivation is found in Appendix B)

$$
\left(\nabla \varphi^{\operatorname{tr}} \cdot \nabla \rho\right)+\nabla^{2} \varphi^{\operatorname{tr}} \rho-2 \omega \mu_{0}=0
$$

where $\rho=1 / \sigma$ (resistivity).

Eq. [14] is the governing equation of this study, which is in the form of a convection-reaction equation. It includes the gradients of conductivity, and is valid for any transmit and receive coil combination. If $\nabla \rho$ is assumed to be zero (which is the case for piecewise homogenous medium approximation), Eq. [14] reduces to the previously proposed phase-based EPT approach (36,37). To obtain Eq. [14], we have made following assumptions, which will be discussed throughout the manuscript: (i) $\nabla\left|\mathrm{B}_{1}^{+}\right|=0$ and $\nabla\left|\mathrm{B}_{1}^{-}\right|=0$; (ii) terms with $\mathrm{B}_{\mathrm{z}}$ are neglected; and (iii) $\sigma^{2} \gg(\omega \epsilon)^{2}$. Finally, a similar derivation can also be made for $\epsilon$, but it is not covered within this manuscript.

\section{METHODS}

\section{Solution of the Central Equation}

Eq. [14] is solved for $\rho$ using the finite-difference method. We do not have to generate a grid for the given geometry, as the measured transceive phase, $\varphi^{\text {tr }}$, is already on the Cartesian grid. Therefore, one can directly represent the partial derivatives with the central finitedifference expressions. For the $(\mathrm{N} \times \mathrm{M} \times \mathrm{L})$ image matrix, the finite-difference formulation of Eq. [14] at a grid point $\left(\mathrm{x}_{\mathrm{i}}, \mathrm{y}_{\mathrm{j}}, \mathrm{z}_{\mathrm{k}}\right)$ is written as

$$
\begin{aligned}
& \left(\left(\left(\frac{\rho_{\mathrm{i}+1, \mathrm{j}, \mathrm{k}}-\rho_{\mathrm{i}-1, \mathrm{j}, \mathrm{k}}}{2 \Delta \mathrm{y}}\right) \cdot \frac{\partial \varphi^{\mathrm{tr}}}{\partial \mathrm{y}}\right)+\left(\left(\frac{\rho_{\mathrm{i}, \mathrm{j}+1, \mathrm{k}}-\rho_{\mathrm{i}, \mathrm{j}-1, \mathrm{k}}}{2 \Delta \mathrm{x}}\right) \cdot \frac{\partial \varphi^{\mathrm{tr}}}{\partial \mathrm{x}}\right)\right) \\
& \quad+\left(\left(\frac{\rho_{\mathrm{i}, \mathrm{j}, \mathrm{k}+1}-\rho_{\mathrm{i}, \mathrm{j}, \mathrm{k}-1}}{2 \Delta \mathrm{z}}\right) \cdot \frac{\partial \varphi^{\mathrm{tr}}}{\partial \mathrm{z}}\right) \\
& \quad+\rho_{\mathrm{i}, \mathrm{j}, \mathrm{k}}\left(\frac{\partial^{2} \varphi^{\mathrm{tr}}}{\partial \mathrm{x}^{2}}+\frac{\partial^{2} \varphi^{\mathrm{tr}}}{\partial \mathrm{y}^{2}}+\frac{\partial^{2} \varphi^{\mathrm{tr}}}{\partial \mathrm{z}^{2}}\right)=2 \omega \mu_{0}
\end{aligned}
$$

where $i=1,2 \cdots \mathrm{N}, j=1,2 \cdots \mathrm{M}, k=1,2 \cdots \mathrm{L}$, and $\Delta \mathrm{x}$, $\Delta y$, and $\Delta z$ are the spatial resolutions in $x, y$, and $z$ directions, respectively. Here, $\rho$ values are the unknowns, and the first and second derivatives of $\varphi^{\text {tr }}$ are the known quantities. $z$ is taken as the slice selection direction.

Before reconstruction, we need to choose the region of interest (ROI) in which the finite-difference formulation is made. For example, we chose a region that has $\mathrm{T}$ spatial grid points, in which $\mathrm{B}$ number of points are the boundary nodes, and $\mathrm{P}=\mathrm{T}-\mathrm{B}$ number of points are the inner nodes. Next, Eq. [15] is written for all P nodes and transformed into $\mathrm{Ax}=\mathrm{b}$ form as

$$
\left[\begin{array}{ccccc}
\mathrm{a}_{11} & \cdots & \mathrm{a}_{1 \mathrm{~B}} & \cdots & \mathrm{a}_{1 \mathrm{~T}} \\
\vdots & \cdots & \vdots & \cdots & \vdots \\
\mathrm{a}_{\mathrm{B} 1} & \cdots & \mathrm{a}_{\mathrm{BB}} & \cdots & \mathrm{a}_{\mathrm{BT}} \\
\vdots & \cdots & \vdots & \cdots & \vdots \\
\mathrm{a}_{\mathrm{T} 1} & \cdots & \mathrm{a}_{\mathrm{TB}} & \cdots & \mathrm{a}_{\mathrm{TT}}
\end{array}\right]\left[\begin{array}{c}
\rho_{1} \\
\vdots \\
\rho_{\mathrm{B}} \\
\vdots \\
\rho_{\mathrm{T}}
\end{array}\right]=\left[\begin{array}{c}
2 \omega \mu_{0} \\
\vdots \\
2 \omega \mu_{0} \\
\vdots \\
2 \omega \mu_{0}
\end{array}\right]
$$

where $\rho_{1}, \rho_{2} \cdots, \rho_{\mathrm{B}}$ are the resistivity values on the boundary and $\rho_{\mathrm{B}+1}, \rho_{\mathrm{B}+2} \cdots, \rho_{\mathrm{T}}$ are the unknown resistivity values. By applying the Dirichlet boundary condition 


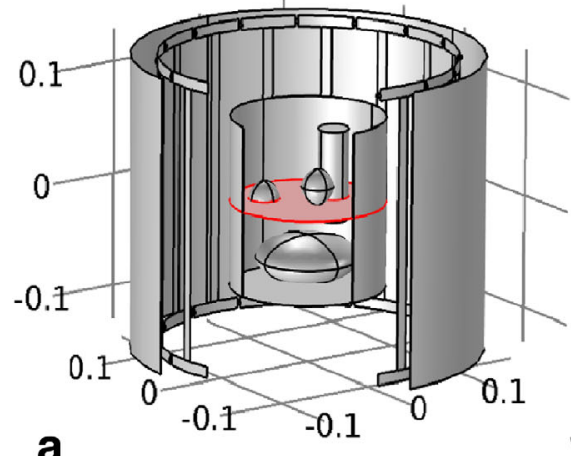

a
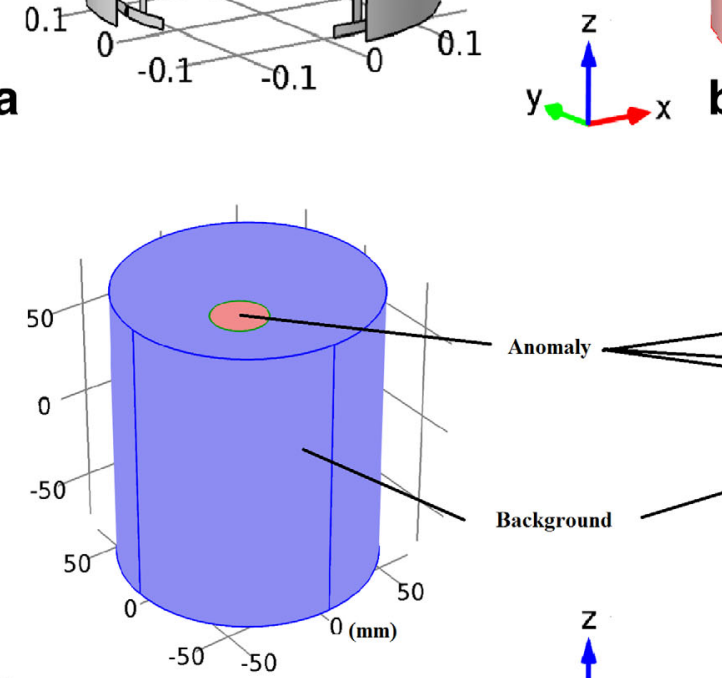

C

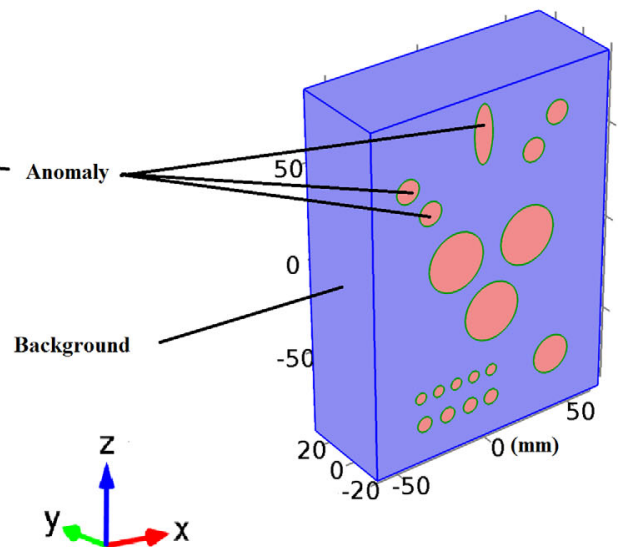

FIG. 1. Birdcage coil simulation models: (a) loaded with the simple phantom; (b) loaded with the head phantom. Experimental phantom models: (c) with one anomaly (left) and with multiple anomalies (right).
( $\rho$ values at the boundary are known), the corresponding columns and rows of $\mathrm{A}$ matrix are eliminated and the resulting matrix, $A_{\mathrm{P} \times \mathrm{P}}$, is used to solve for $\rho_{\mathrm{B}+1}, \rho_{\mathrm{B}+2} \cdots$, $\rho_{\mathrm{T}}$ values. Here, transformation from the matrix index $\left(\rho_{\mathrm{i}, \mathrm{j}, \mathrm{k}}\right)$ used in Eq. [15] to the linear index $\left(\rho_{1}, \rho_{2} \cdots, \rho_{\mathrm{T}}\right)$ used in Eq. [16] is not explicitly given, but it is straightforward.

Eq. [14] is in the form of the general convectiondiffusion-reaction equation. In this equation, $\left(\nabla \varphi^{\operatorname{tr}} \cdot \nabla \rho\right)$ is the convection term, $\left(\nabla^{2} \varphi^{\mathrm{tr}} \rho-2 \omega \mu_{0}\right)$ is the reaction term, and there is no diffusion term. The numerical solution of this equation is a challenge if the convection term dominates the diffusion term, and the solution will have unwanted spurious oscillations near the interior and boundary layers $(41,42)$. In our case, because the system is purely convective (there is no diffusion term), we expect these oscillations in our solutions. To solve this issue, we added an artificial diffusion term to Eq. [14], which stabilizes the solution without blurring the internal layers significantly. This method is one of the widely used stabilization methods, and it is easy to implement (41,42). After adding an artificial diffusion term, Eq. [14] becomes

$$
-c \nabla^{2} \rho+\left(\nabla \varphi^{\mathrm{tr}} \cdot \nabla \rho\right)+\nabla^{2} \varphi^{\mathrm{tr}} \rho-2 \omega \mu_{0}=0
$$

where $c$ is the constant diffusion coefficient. Similar to the convection and reaction terms, the diffusion terms, $\left(-c \nabla^{2} \rho\right)$, can be discretized using three-point central difference approximation, and be added to Eq. [15]. The final matrix equation is solved using MATLAB (back- slash operator). Because the final matrix is square and of full rank, MATLAB finds $\mathrm{A}^{-1} \mathrm{~b}$ using Gaussian elimination without explicitly finding $\mathrm{A}^{-1}$, thereby providing speed. Adding a diffusion term as shown in Eq. [17] significantly improves the condition number of system matrix A. For example, for the reconstruction shown in Figure 5, the condition number of $\mathrm{A}$ is equal to 1833 and 154 without and with the diffusion term $(\mathrm{c}=0.05)$, respectively.

To decrease the matrix size and make the computations faster, Eq. [14] and subsequently Eq. [17] can be reduced to two-dimensional (2D) form in some cases, where, eg, $\partial \rho / \partial z$ or $\partial \varphi^{\text {tr }} / \partial z$ is negligible in the region of interest.

\section{Simulation Methods}

Electromagnetic simulations were performed using COMSOL Multiphysics 4.2a (COMSOL AB, Stockholm, Sweden), and the simulated data were exported to MATLAB (The Mathworks, Natick, Massachusetts), where the reconstruction algorithm was implemented.

In the simulations, quadrature RF birdcage coil was modeled and loaded with the simple phantom model or the human head model shown in Figures 1a and 1b (43). The simulations were made at $128 \mathrm{MHz}(3 \mathrm{~T})$ with a voxel size of $2 \times 2 \times 2 \mathrm{~mm}^{3}$. The conductivity maps were calculated using the simulated transceive phase, which is acquired by the summation of $\mathrm{B}_{1}^{+}$and $\mathrm{B}_{1}^{-}$phases of the coil. The transmit magnetic field was computed as $\mathrm{B}_{1}^{+}=\left(\mathrm{B}_{\mathrm{x}}+\mathrm{iB}_{\mathrm{y}}\right) / 2$, and the receive magnetic field was calculated as $\mathrm{B}_{1}^{-}=\left(\mathrm{B}_{\mathrm{x}}-\mathrm{iB}_{\mathrm{y}}\right) / 2$. A comparison was made 
Table 1

Parameters of the Balanced SSFP Sequences for Each Experiment

\begin{tabular}{|c|c|c|c|c|c|c|c|}
\hline Experiment & Resolution (mm) & FOV (mm) & Orientation & FA (deg) & $\mathrm{TR} / \mathrm{TE}(\mathrm{ms})$ & NEX & Duration (min) \\
\hline Phantom with an anomaly & $1.56 \times 1.56 \times 5$ & $200 \times 200 \times 5$ & Transverse 2D & 60 & $4.18 / 2.09$ & 32 & $\sim 0.5$ \\
\hline $\begin{array}{l}\text { Phantom with multiple } \\
\text { anomalies }\end{array}$ & $1.56 \times 1.56 \times 1.56$ & $200 \times 200 \times 16$ & Coronal 3D & 40 & $4.9 / 2.45$ & 32 & $\sim 8.5$ \\
\hline Human brain & $1.7 \times 1.7 \times 1.7$ & $220 \times 220 \times 190$ & Sagittal 3D & 45 & $4.42 / 2.21$ & 10 & $\sim 9.5$ \\
\hline
\end{tabular}

between the conventional phase-based EPT method that uses the formula $\sigma=\nabla^{2} \varphi^{\text {tr }} / 2 \omega \mu_{0}$, and the proposed method that uses Eq. [17] for noisy simulated data. The noise distribution in MRI phase images is assumed to be zero-mean Gaussian with a standard deviation of $1 /(\sqrt{2} \mathrm{SNR})$, in which SNR is the signal-to-noise ratio in MR magnitude images $(44,45)$. In the simulations, SNR values 100, 200, 400, and $\infty$ were employed for each method, and the performance of these methods against noisy data was investigated.
The total relative error in the reconstructed conductivity images are calculated using the $L^{2}$ norm as

$$
\mathrm{E}_{\sigma}=\sqrt{\frac{\sum_{k=1}^{P}\left(\sigma_{k, a}-\sigma_{k}\right)^{2}}{\sum_{k=1}^{P} \sigma_{k, a}{ }^{2}}}
$$

where $\sigma_{k, a}$ and $\sigma_{k}$ are the actual and reconstructed conductivity values for the $k^{\text {th }}$ node, respectively.
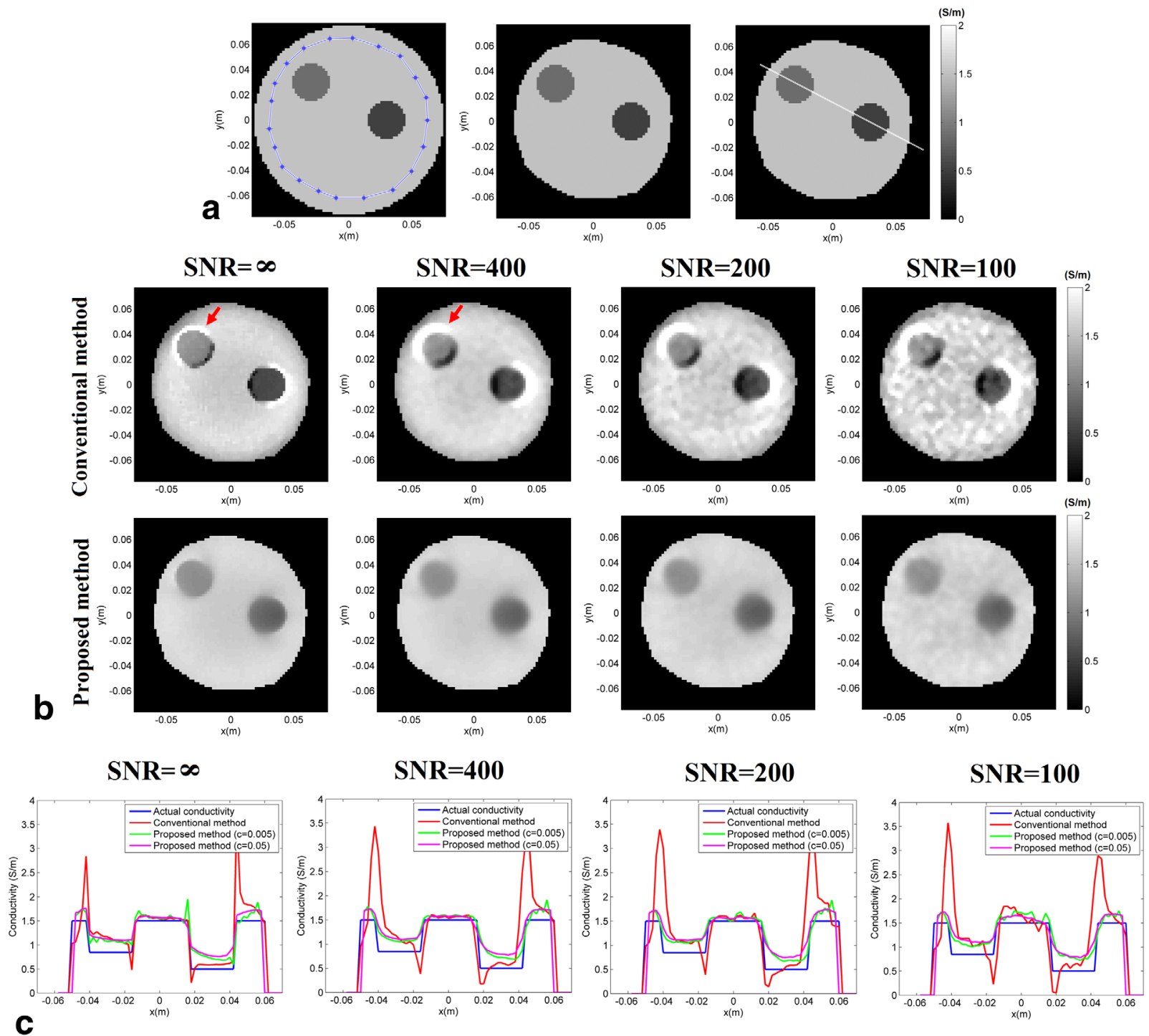

FIG. 2. (a) Selection of the ROI indicated by the blue polygon (left), the actual conductivity map in the ROI (middle), and illustration of the line where the conductivity profiles are plotted (right). (b) Reconstructed conductivity maps using the conventional phase-based EPT method and the proposed method for different SNR values. (c) Conductivity profiles of the conventional method and the proposed method (along the dotted line given in (a)) for different diffusion coefficients under different SNR values. 

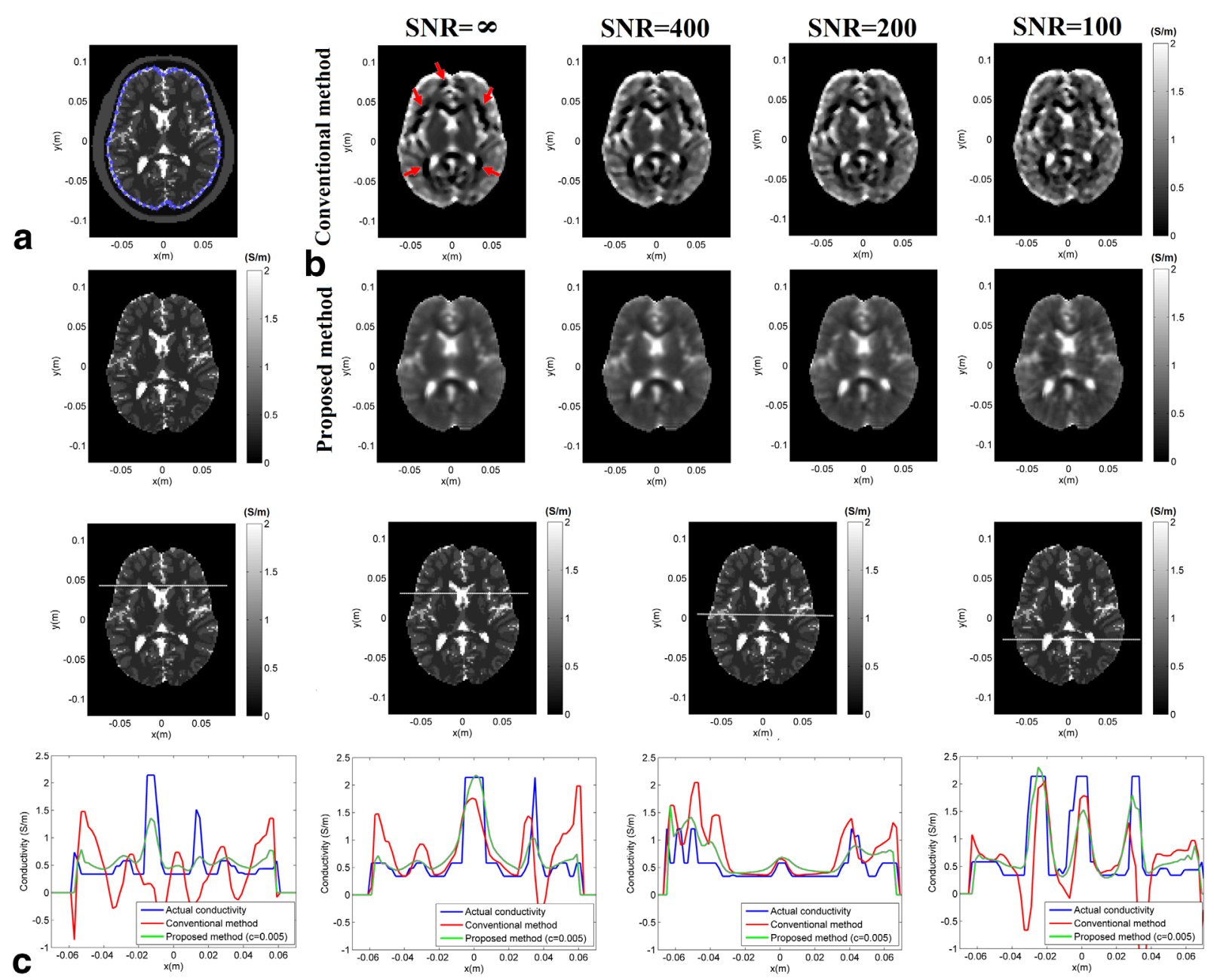

FIG. 3. Human head model simulations: (a) selection of the ROI indicated by the blue polygon (left), the actual conductivity map in the $\mathrm{ROI}$ (right); (b) reconstructed conductivity maps using the conventional phase-based EPT method and the proposed method for different SNR values; (c) conductivity profiles of the conventional method and the proposed method along the lines that are shown above each profile plot (when the $\mathrm{SNR}=\infty$ ).

\section{Experimental Methods}

\section{Phantom Setup}

Two different experimental phantoms (described in Fig. 1c) were constructed. The background region of the phantoms was made using an agar-saline solution (20 g/L Agar, $2.5 \mathrm{~g} / \mathrm{L} \mathrm{NaCl}, 0.2 \mathrm{~g} / \mathrm{L} \mathrm{CuSO}_{4}$ ), and the higher conductivity (anomaly) regions were prepared using a saline solution ( $8.8 \mathrm{~g} / \mathrm{L} \mathrm{NaCl}, 0.2 \mathrm{~g} / \mathrm{L} \mathrm{CuSO}_{4}$ ). The conductivity values of the background and the anomaly regions are expected to be approximately 0.55 and $1.5 \mathrm{~S} / \mathrm{m}$, respectively (46). A conductivity meter (8733, Hanna Instruments, Woonsocket, Rhode Island) was also used to measure the con- ductivity of the saline solution and was found to be 1.53 $\mathrm{S} / \mathrm{m}$. For the agar-saline solutions, the effect of agar to the conductivity was taken into consideration as given in (47), and the agar is assumed to contribute an additional conductivity of $0.1 \mathrm{~S} / \mathrm{m}$. The relative dielectric permittivity of the phantom compartments, which have different $\mathrm{NaCl}$ concentrations, are expected to be approximately 80, which is the same as the salt-free water. It is stated in (46) that the dielectric permittivity of a saline solution is not different from the dielectric permittivity of a salt-free water in the frequency range of $10 \mathrm{~Hz}$ to $200 \mathrm{MHz}$. The effect of agar to dielectric permittivity is negligible at the frequency of interest (46).

Table 2

Total Relative Errors in the Reconstructed Conductivity Maps

\begin{tabular}{|c|c|c|c|c|c|c|c|c|}
\hline \multirow[b]{2}{*}{ Simulation } & \multicolumn{2}{|c|}{$\mathrm{SNR}=\infty$} & \multicolumn{2}{|c|}{$\mathrm{SNR}=400$} & \multicolumn{2}{|c|}{$\mathrm{SNR}=200$} & \multicolumn{2}{|c|}{$\mathrm{SNR}=100$} \\
\hline & $\mathrm{CM}$ & PM & $\mathrm{CM}$ & PM & $\mathrm{CM}$ & PM & $\mathrm{CM}$ & PM \\
\hline Phantom & $8.92 \%$ & $2.18 \%$ & $9.77 \%$ & $2.23 \%$ & $10.1 \%$ & $2.31 \%$ & $10.5 \%$ & $2.47 \%$ \\
\hline Human head & $73.71 \%$ & $16.36 \%$ & $74 \%$ & $16.4 \%$ & $74.69 \%$ & $16.65 \%$ & $76.78 \%$ & $17.47 \%$ \\
\hline
\end{tabular}

CM, conventional method; PM, proposed method. 
FIG. 4. Magnitude, phase, and reconstructed conductivity images of the first experimental phantom for one of the channels in the ROI: (a) SSFP magnitude image; (b) SSFP transceive phase; (c) conductivity map reconstructed using the conventional method; (d) conductivity map. reconstructed using the proposed method.
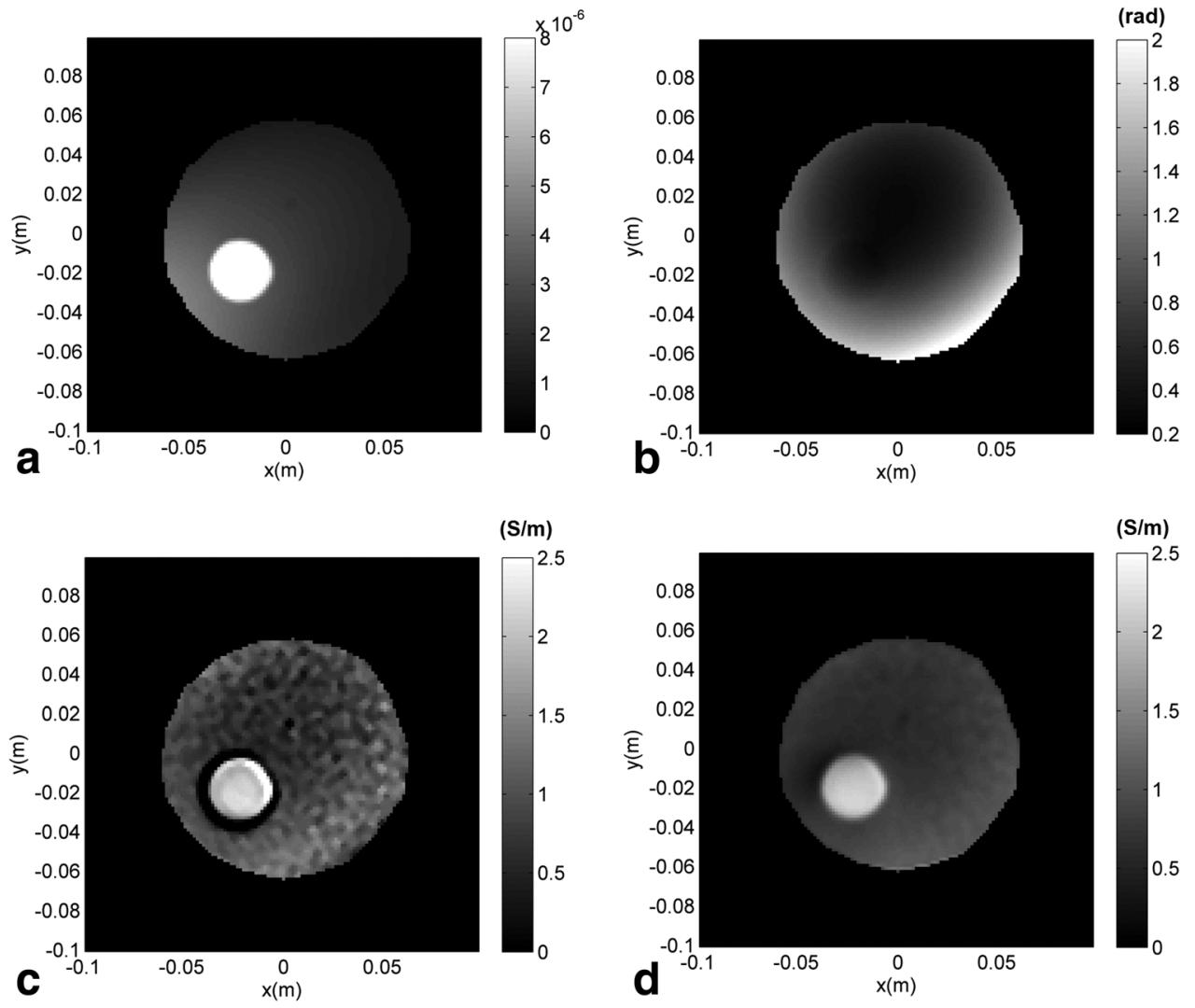

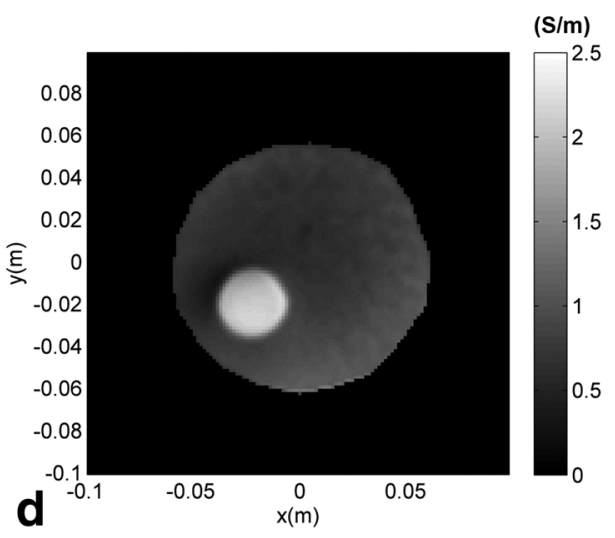

\section{In Vivo Human Experiment}

A healthy male volunteer (age 23 years) was also studied with the approval of the Institutional Review Board of Bilkent University. Electrical conductivity maps in the brain were reconstructed using the proposed method.

\section{Sequence Protocols and Reconstruction}

All experiments were conducted on a 3T Magnetom Trio MR Scanner (Siemens, Erlangen, Germany), installed in UMRAM (National Magnetic Resonance Research Center) at Bilkent University. Standard quadrature body coil was used for transmit and 12-channel receive-only phased array head coil was used for receive in both the phantom and human experiments. To measure the transceive phase, a balanced steadystate free-precession (SSFP) sequence was applied. The sequence parameters of each experiment are given in Table 1.

To reduce the edge ringing artifact in SSFP magnitude and phase images, a reconstruction filter (Hamming window) was applied to the k-space data. For the boundary condition, all boundary nodes were assigned the same value, which is $1.5 \mathrm{~S} / \mathrm{m}$ for the simulations and for the human experiment, and $0.5 \mathrm{~S} / \mathrm{m}$ for the experimental phantoms. For the diffusion coefficient introduced in Eq. [17], different values from 0.005 to 0.05 are used. For noisy simulations and the experiments, the Gaussian filter with a kernel size of $5 \times 5 \times 5$ voxels and a standard deviation of 1.06 for each direction was applied to the transceive phase data. Additionally, a median filter with a kernel size of $3 \times 3 \times 3$ voxels was applied to the conductivity maps of the conventional phase-based EPT method to obtain smoother reconstruction results (48).
In the simulations, because the birdcage coil is used for both transmit and receive, $\partial \varphi^{\text {tr }} / \partial \mathrm{z}$ is negligibly small when compared with $\partial \varphi^{\mathrm{tr}} / \partial \mathrm{x}$ and $\partial \varphi^{\mathrm{tr}} / \partial \mathrm{y}$. Additionally, for the first and second experimental phantoms, because the conductivity does not change in the slice-selection direction, $\partial \rho / \partial z$ is negligibly small. For these cases, therefore, conductivity maps were obtained using the $2 \mathrm{D}$ form of the proposed method. However, for the human experiment, the conductivity maps were obtained using the three-dimensional (3D) form.

For the reconstructions, a HP Z800 workstation with Intel Zeon X5675 3.07 GHz dual processors (12 cores) and with $64 \mathrm{~GB}$ of RAM was used. The computation time of $2 \mathrm{D}$ reconstruction of the conductivity maps for the $128 \times 128$ image matrix was approximately $0.8 \mathrm{~s}$. The computation time of the 3D human experiment reconstructions was approximately $67 \mathrm{~s}$ for the $128 \times 128 \times 9$ image matrix.

\section{RESULTS}

\section{Simulation Results}

Figure 2 shows the reconstructed conductivity maps of the simulation phantom (Fig. 1a). It is observed that the conventional method reconstructs the conductivity accurately in the regions where the electrical properties do not vary, but it yields an artifact in the transition regions, which are shown by the red arrows in the first row of Figure $2 \mathrm{~b}$. This boundary artifact gets wider when the Gaussian filter is used in the noisy simulations. In contrast, the proposed method calculates the conductivity maps successfully in the whole ROI, including the transition regions (second row of Fig. 2b). The superiority of the 

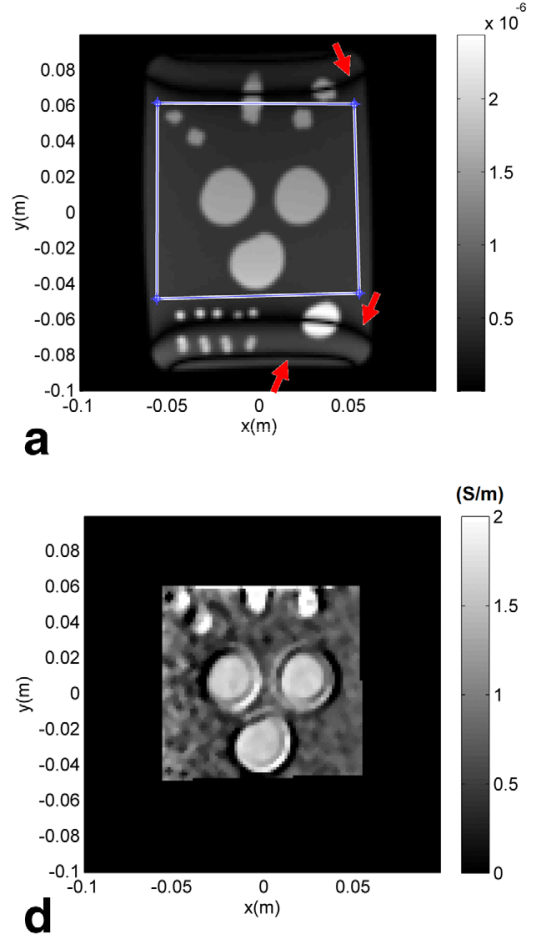
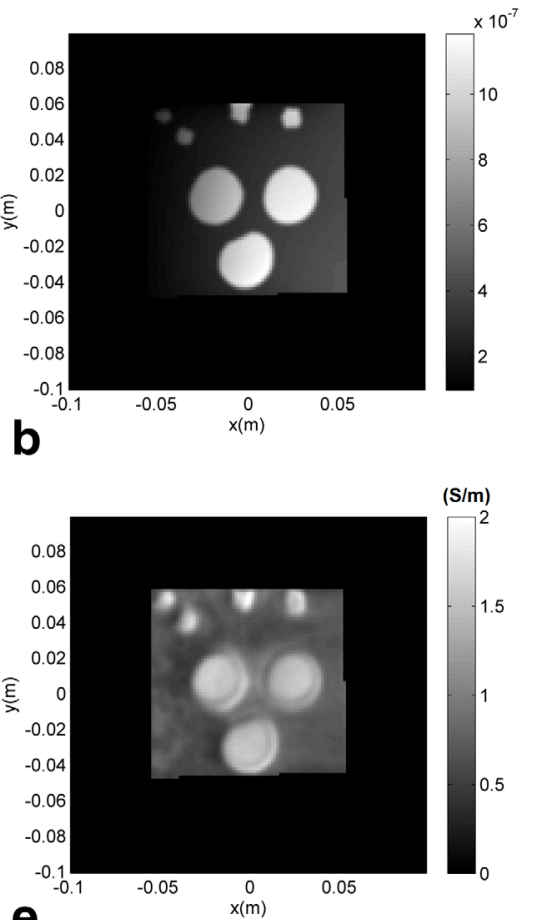

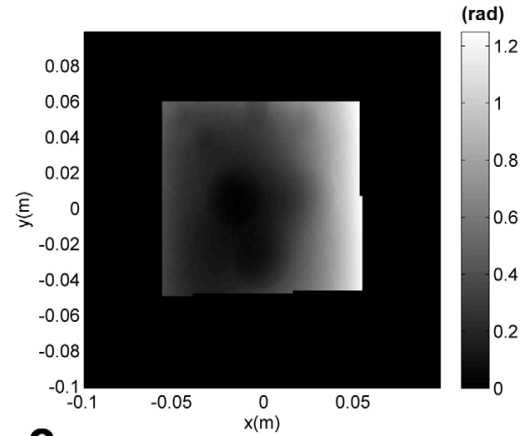

C

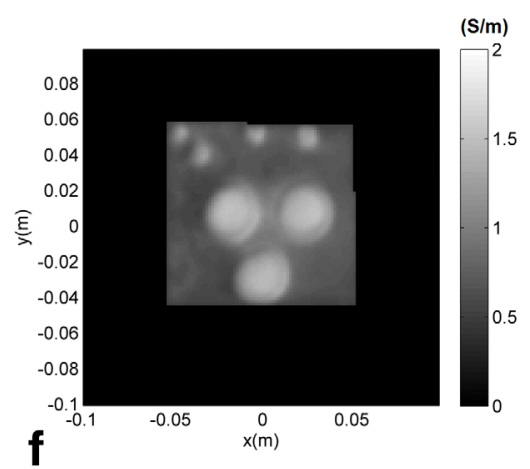

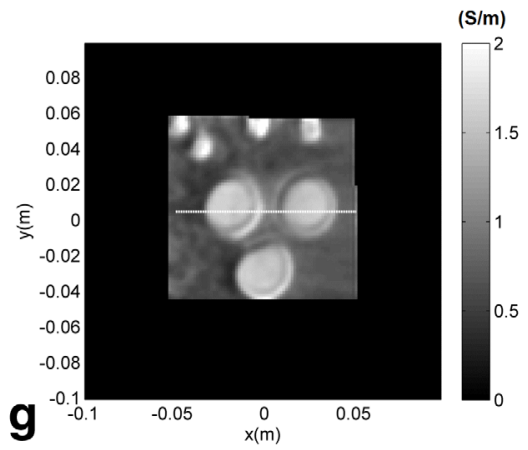

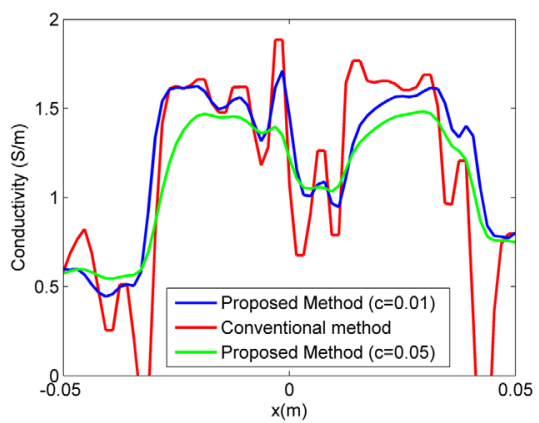

FIG. 5. Magnitude, phase, and reconstructed conductivity images of the second experimental phantom for one of the channels: (a) selection of the ROI indicated by the blue polygon; (b) SSFP magnitude image; (c) SSFP transceive phase; (d) conductivity map using the conventional method; (e) conductivity map using the proposed method (c=0.01); (f) conductivity map using the proposed method $(\mathbf{c}=0.05)$; (g) conductivity profiles of the conventional and the proposed method given in (d)-(f).

proposed method over the conventional method is more apparent in the conductivity profiles shown in Figure 2c. The effect of using different diffusion coefficients $(0.05$ and 0.005 ) in the proposed method can be also seen in Figure 2c. The use of a smaller diffusion coefficient allows for the calculation of the conductivity to be more accurate, but in a less stable manner. In other words, there is a tradeoff between the accurateness and the stabilization of the solution when choosing the diffusion coefficient in the proposed method.

Figure 3 shows the reconstructed conductivity maps for the human head simulation with diffusion constant, $\mathrm{c}=0.005$. In the reconstructed conductivity maps of the conventional method, dark regions, which are shown with the red arrow in Figure $3 \mathrm{~b}$, are the boundary artifacts. These regions are well reconstructed in the proposed method, and are shown in the second row of Figure $3 \mathrm{~b}$.

The total relative errors in the calculated conductivity maps of both methods are given in Table 2. Errors in the human head simulation are higher than the errors in the phantom simulation. In the conventional method, the main reason for this is the complexity of the structure. By complexity, we mean that the geometry has many transition boundaries. The more complex structures we have in the region of interest, the more boundary artifacts occur in the conventional method. This yields a significant error in the reconstructed conductivity maps. In contrast, in the proposed method, the main source of the error is the violation of the assumption of $\sigma^{2} \gg(\omega \epsilon)^{2}$. Especially in the low conductive regions of the brain, ie, the white matter (with literature values $(51), \sigma \approx 0.35 \frac{\mathrm{s}}{\mathrm{m}}$, $\epsilon_{r} \approx 53$ at $128 \mathrm{MHz}$ ) and the gray matter (with literature values (51), $\sigma \approx 0.58 \frac{\mathrm{s}}{\mathrm{m}}, \epsilon_{r} \approx 75$ at $128 \mathrm{MHz}$ ), the conductivity values are found to be $0.46 \pm 0.01 \mathrm{~S} / \mathrm{m}$ and $0.75 \pm 0.02 \mathrm{~S} / \mathrm{m}$, respectively. These overestimated values are the result of the violation of the assumption at the frequency of interest ( $>100 \mathrm{MHz}$ ). However, the error in the proposed method is still acceptable when compared with the conventional method. Figure 3c shows the conductivity profiles of both methods for different lines in the ROI. 

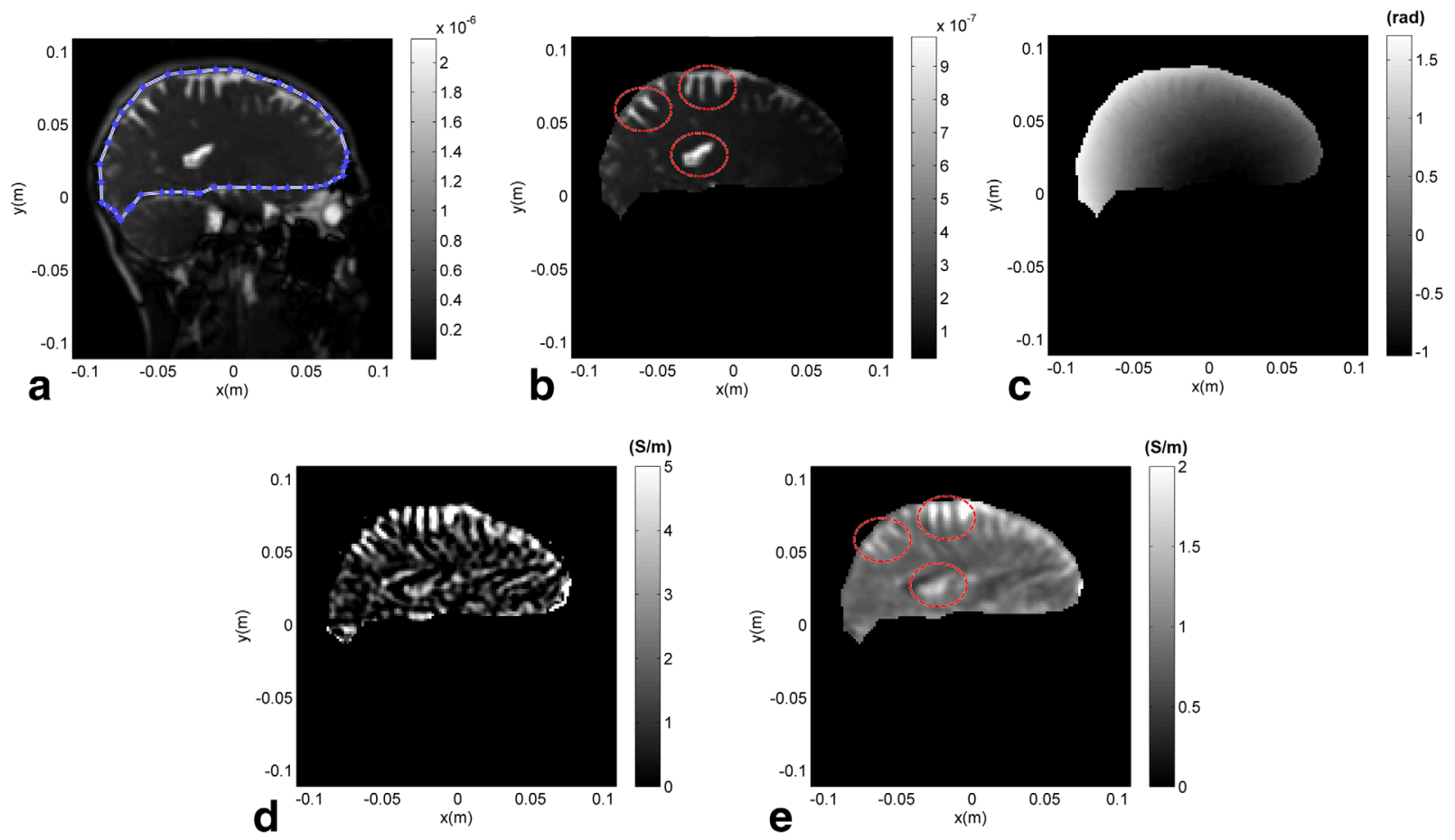

FIG. 6. Magnitude, phase, and reconstructed conductivity images of human brain for one of the channels at the ROI: (a) selection of the $\mathrm{ROI}$ indicated by the blue polygon; (b) SSFP magnitude image; (c) SSFP transceive phase; (d) conductivity map reconstructed using the conventional method; (e) conductivity map reconstructed using the 3D formulation of the proposed method.

For all lines, the conductivity profiles of the proposed method are well consistent with the actual conductivity maps.

\section{Experimental Results}

Figure 4 shows the results for the phantom with one anomaly (described in Fig. 1c). SNR was calculated using two repeated acquisitions (49) and was found to be approximately 125 for the background, and 220 for the anomaly region. With the proposed method $(\mathrm{c}=0.05)$, the conductivity values in the anomaly region and the background region are found to be $1.93 \pm 0.07 \mathrm{~S} / \mathrm{m}$ and $0.6 \pm 0.01 \mathrm{~S} / \mathrm{m}$, respectively. With the conventional method, the conductivity values are found to be $2.07 \pm 0.07 \mathrm{~S} / \mathrm{m}$ and $0.59 \pm 0.09 \mathrm{~S} / \mathrm{m}$. Transition regions, where boundary artifacts occur with the use of the conventional method, were well reconstructed in the proposed method.

Figure 5 shows the results for the more complex phantom (described in Fig. 1c). The SSFP magnitude image is illustrated in Figure 5a. Here, banding artifacts, which can occur in SSFP sequences, are observed, and they are shown with the red arrows. Fixing this banding artifact issue is beyond the scope of this manuscript (interested readers are referred to (50)); therefore, the ROI is selected as shown in Figure 5a. As shown in Figure 5b, sensitivity of this channel (or coil) drops when moving from the lower-right corner to the upper-left corner. For the more sensitive sides, SNR was calculated to be approximately 200 for the anomaly region and 100 for the background region. For the less sensitive sides, SNR was calculated to be approximately 90 for the anomaly region and 50 for the background region. It is observed in Figures 5e-5g that the proposed method successfully reconstructs the transition regions in the complex phantom, which has small anomalies (with radii of less than $1 \mathrm{~cm}$ ).

Figure 6 shows in vivo results in a sagittal slice of the human volunteer. The multichannel combined SSFP magnitude image, and the selection of the ROI, are shown in Figure 6a. SNR in the CSF regions was found to be 400, and for non-CSF regions it was found to be 90. Figures $6 \mathrm{~d}$ and 6 e show the results of the conductivity maps at a sagittal slice of the conventional method and the proposed method (using 3D formulation), respectively. The 3D region was selected by taking four slices above and below the sagittal slice, and the final matrix $(128 \times 128 \times 9)$ was used to solve the conductivity. In the results of the conventional method, dark regions that have negative conductivity values are the boundary artifacts, and it is difficult to interpret this image for any clinical purposes. In contrast, with the proposed method ( $\mathrm{c}=0.05)$, the boundary artifacts are eliminated. With the proposed method, the average conductivity values of the CSF regions in the red dotted circles (Figs. 6b and 6e) are found to be $2.08 \pm 0.3 \mathrm{~S} / \mathrm{m}, 1.4 \pm 0.09 \mathrm{~S} / \mathrm{m}$, and $1.66 \pm 0.04 \mathrm{~S} / \mathrm{m}$, from upper to lower side. The average conductivity value of the gray matter (which lies primarily adjacent to the CSF regions in Fig. 6d) is found to be $0.76 \pm 0.03 \mathrm{~S} / \mathrm{m}$, and for the white matter (calculated in the central region in Fig. 6d) the conductivity value is found to be $0.53 \pm 0.06 \mathrm{~S} / \mathrm{m}$. For the low conductive regions (ie, gray matter and the white matter), the conductivity values are overestimated similar to the simulation results.

\section{DISCUSSION}

In this study, a boundary artifact-free electrical conductivity imaging method based solely on the MR transceive 
$B C=0.5 \mathrm{~S} / \mathrm{m}$
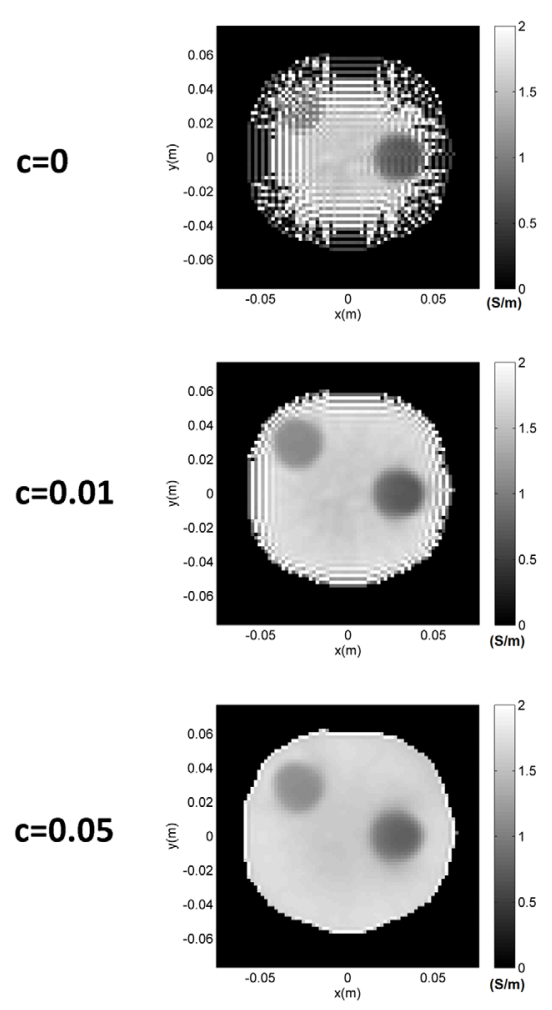

$B C=1 \mathrm{~S} / \mathrm{m}$
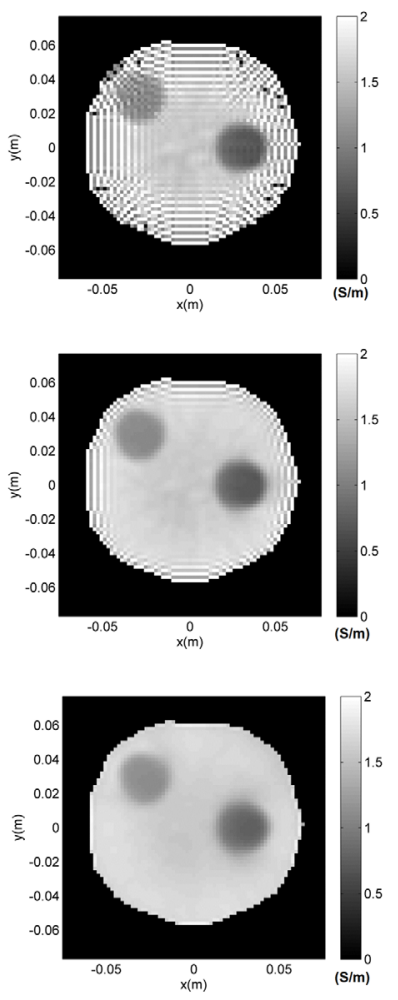

$B C=1.5 \mathrm{~S} / \mathrm{m}$
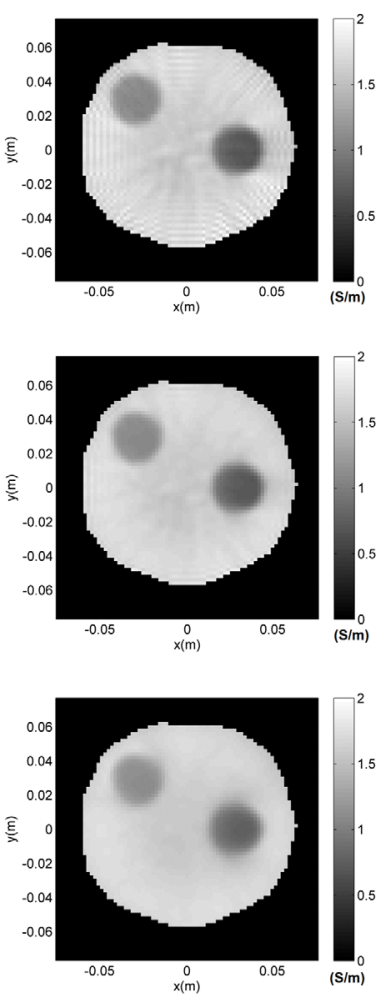
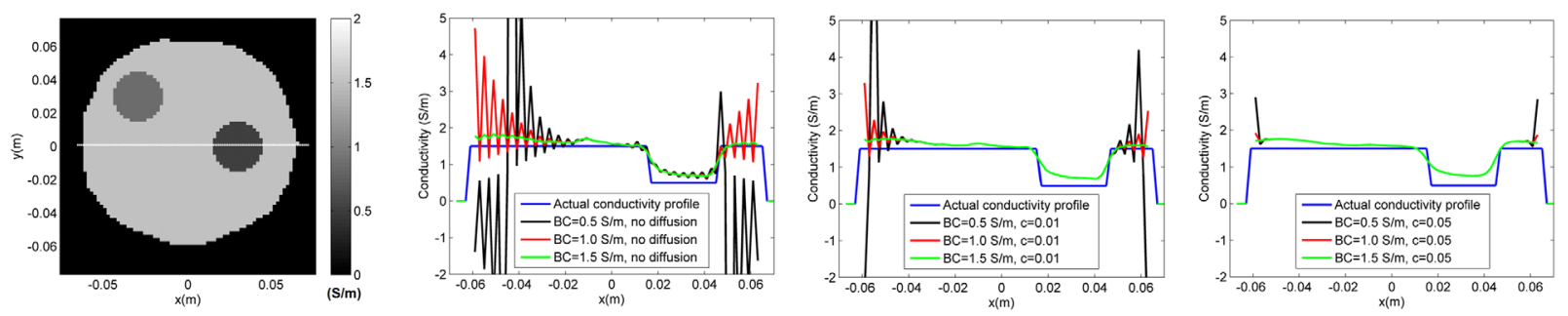

FIG. 7. Illustration of the oscillatory decay from the given boundary (initial) value to the final value under different diffusion coefficients ( $\mathrm{c}=$ diffusion coefficient) and boundary conditions $(\mathrm{BC}=$ value of the conductivity assigned at the boundary). Background conductivity of the simulation phantom is $1.5 \mathrm{~S} / \mathrm{m}$. For the worst case $(\mathrm{c}=0, \mathrm{BC}=0.5 \mathrm{~S} / \mathrm{m})$, excessive spurious oscillations were observed in the conductivity map; however, oscillations decrease as the BC approaches to the exact value of the background conductivity. For $\mathrm{c}=0.01$, these oscillations were significantly reduced. For $\mathrm{c}=0.05$, the effect of wrong $\mathrm{BC}$ is confined to a few pixels only. The results are also shown in the profile plots for better visualization.

phase has been proposed. Different from the previous phase-based EPT approach $(36,37)$, a new formulation, which is in the form of a convection reaction equation, was derived without assuming that the gradient of the conductivity is zero. With this contribution, this study resolves the boundary artifact issue of the conventional phase-based EPT, and paves the way for fast and reliable reconstruction of the conductivity maps of tissues in clinical applications.

There are two significant advantages of the proposed method. One is the noise reduction ability when solving the governing equation. This comes from the use of the diffusion term $\left(-c \nabla^{2} \rho\right)$, which acts as a low-pass filter in the solutions without significantly blurring the final conductivity maps. The noise performance of the pro- posed method and the conventional method can be compared in Figures 2 and 3. The second advantage of the proposed method is the ability to successfully reconstruct the transition regions where boundary artifacts (overshoot or undershoot in the conductivity maps) occur in the conventional method. Especially for practical applications, because of the use of strong spatial filters to reduce the noise in the phase data, the boundary artifact in the conventional method becomes wider. For complex structures (eg, brain), this leads to unreliable conductivity maps, which are difficult to interpret for clinical diagnosis.

To derive the governing equation of the proposed method, three assumptions have been made. One is $\nabla\left|\mathrm{B}_{1}^{+}\right|=0$ and $\nabla\left|\mathrm{B}_{1}^{-}\right|=0$, which state that the magnitude 

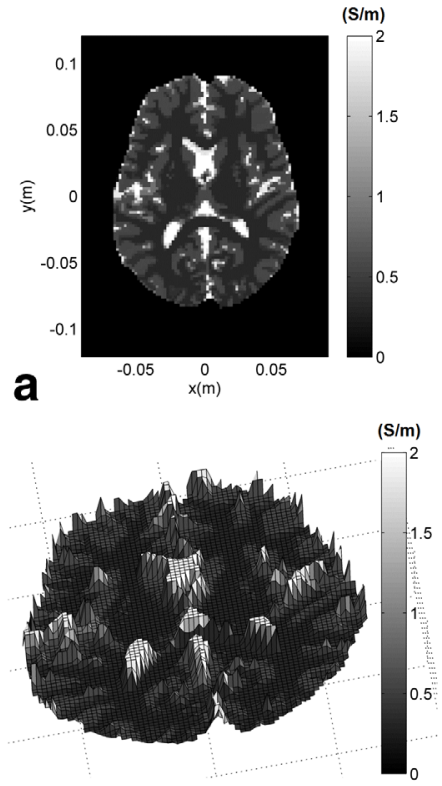
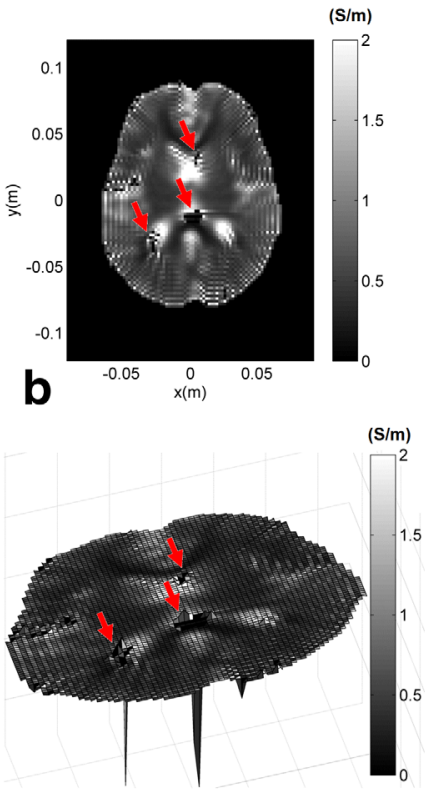
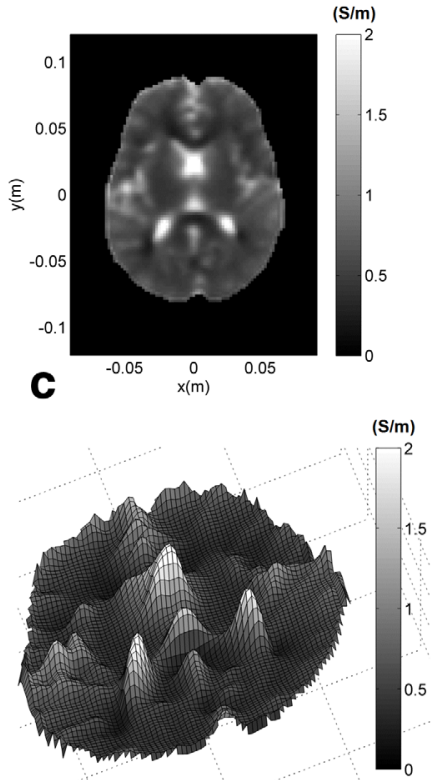
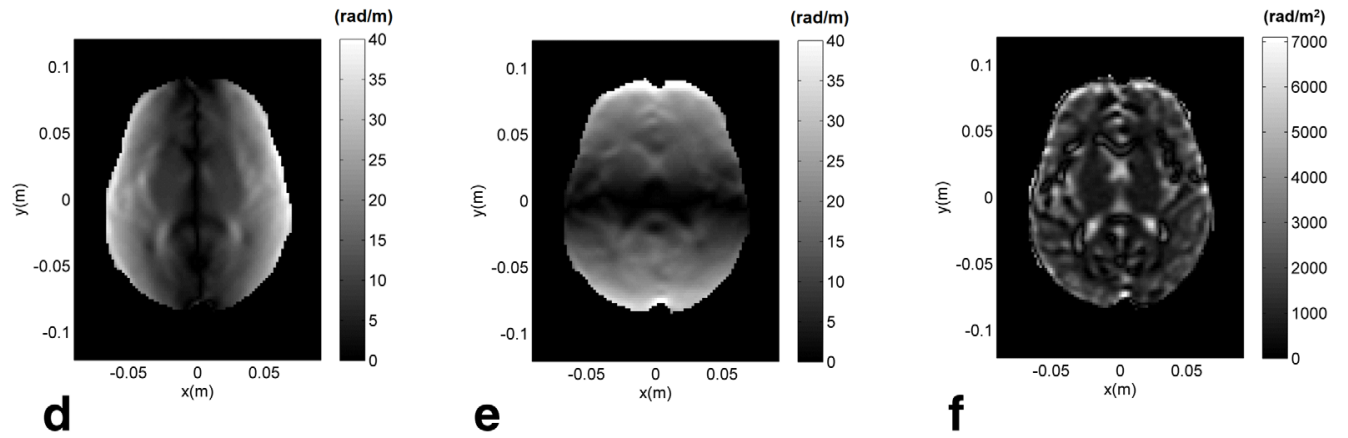

FIG. 8. Reconstructed conductivity maps of simulated human brain for different diffusion coefficients. (a) 2D and 3D surface plots of the actual conductivity of an axial slice; (b) $2 \mathrm{D}$ and $3 \mathrm{D}$ surface plots of reconstructed conductivity of the same slice for $\mathrm{C}=0$; (c) same as in (b) for $\mathrm{c}=0.05$; (d) magnitude of the $\mathrm{x}$ component of the gradient of the transceive phase, $\left|\frac{\partial}{\partial x} \varphi^{\mathrm{tr}}\right|$; (e) magnitude of the $y$ component of the gradient of the transceive phase, $\left|\frac{\partial}{\partial y} \varphi^{\mathrm{tr}}\right|$; (f) magnitude of the Laplacian of the transceive phase, $\left|\nabla^{2} \varphi^{\mathrm{tr}}\right|$. In the regions where $\left|\frac{\partial}{\partial x} \varphi^{\mathrm{tr}}\right|,\left|\frac{\partial}{\partial y} \varphi^{\mathrm{tr}}\right|$, and $\left|\nabla^{2} \varphi^{\mathrm{tr}}\right|$ are close to zero, spot-like artifacts are observed and are shown with the red arrows. The use of the diffusion term $(\mathrm{c}=0.05)$ prevents these high variations in the reconstructions and make the solutions more stable.

of the transmit and receive magnetic fields in the ROI vanish (or vary slowly). This assumption starts to fail at high field strengths $(>3 \mathrm{~T})$. It has been shown that $\mathrm{RF}$ shimming can be used to improve the conventional phase-based conductivity maps by modifying the $\mathrm{B}_{1}^{+}$field in the ROI (52), which can also be used in the proposed method. The second assumption is $\sigma^{2} \gg(\omega \epsilon)^{2}$. This assumption is valid for most of the human tissues at field strengths $\leq 3 \mathrm{~T}$. Violation of this assumption (such as in the gray matter and the white matter) causes overestimation of the conductivity (see Figs. 2 and 3). It is shown in (36) that the conventional phase-based EPT method also relies on a similar approximation (namely, $\sigma \gg \omega \epsilon$ ), and overestimated conductivities can also be observed $(36,53)$. The third assumption is that the first derivatives of $\mathrm{B}_{\mathrm{z}}$ are negligible when compared with $\mathrm{B}_{1}^{+}$and $\mathrm{B}_{1}^{-}$. For the birdcage coil (or TEM coil), this assumption is valid at the central region of the coil. However, for different coil configurations, such as transmit from birdcage coil and receive from phased array coil, this assumption may not hold for the regions where the receive coils are less sensitive. Depending on the variation of the tissue EPs in the $\mathrm{z}$-direction, derivatives of $\mathrm{B}_{\mathrm{z}}$ can be comparable with the transverse magnetic field in the low-sensitive regions of the receive coils, and also at the off-center regions of the coils. In such situations, $B_{z}$ can be estimated by integrating the $B_{x}$ and $B_{y}$ using Gauss's Law, in which $B_{x}$ and $B_{y}$ can be approximately found from the determined $\mathrm{B}_{1}^{+}$and $\mathrm{B}_{1}^{-}$(53).

To solve the governing partial differential equation of the proposed method, the Dirichlet boundary condition is applied, that is, the conductivity value on the boundary of the ROI is assigned. It is found that the exact value assigned to the boundary is not critical except for a narrow band around the boundary. In other words, even if the boundary condition is taken as very different from the exact value, the solution immediately decays to the desired value within a few pixels (see Fig. 7). This is because of the use of the diffusion term in the solutions (see Eq. [17]), which acts as a regularization term, and makes the problem more stable by preventing the high variations (including the spurious decaying oscillations) 
in the reconstructed conductivity maps both in the case of outer boundary (see Fig. 7) and in the case of internal boundaries (see Fig. 5g). Hence, this enables the use of the proposed method in any local ROI as presented in (40).

In the cr-MREPT method (25), it was reported that spot-like artifacts occurred in the regions where the magnitude of the convective field was low. A similar artifact can also be observed in the proposed method. With reference to Eq. [14], which does not include a diffusion term, it can be argued that if $\nabla^{2} \varphi^{\text {tr }}$ and $\nabla \varphi^{\text {tr }}$ are close to zero in the same region, huge variations (spot-like artifact) in $\rho$ are allowed in the solution. Such artifacts are shown in Figure 8b. In contrast, if the diffusion term is included as in Eq. [17], high variations in the reconstructions are prevented and the solutions become more stable, as shown in Figure 8c.

Combining the proposed method with the convection reaction-based MREIT method (54), one can image the conductivity simultaneously at frequencies below a few $\mathrm{kHz}$ and at Larmor frequency, such as the recently published hybrid methods $(55,56)$.

\section{CONCLUSIONS}

In summary, a new phase-based electrical conductivity imaging method that includes the electrical conductivity gradient terms has been formulated, and the final partial differential equation has been solved using the finitedifference scheme. The superiorities of the proposed method over the conventional method are the boundary artifact-free reconstruction ability and the robustness against noise. With these two advantages and the inherent advantages of the phase-based EPT (fast, TPA-free, and practically applicable for any transmit-receive coil configuration), the proposed method provides fast and reliable electrical conductivity images for clinical applications and SAR estimation. Application of the proposed method to patient data will be the future research direction.

\section{ACKNOWLEDGMENT}

This study was supported by TUBITAK 114E522 research grant. Experimental data were acquired using the facilities of UMRAM, Bilkent University, Ankara.

\section{APPENDIX A: CONVECTION REACTION-BASED MREPT (cr-MREPT) FORMULA}

Here, a $\mathrm{B}_{1}^{-}$-based cr-MREPT formula in the logarithm form will be given (derivation of the $\mathrm{B}_{1}^{+}$-based cr-MREPT formula has already been given in (25)). We start with writing the $x$ and $y$ components of Eq. [1] as

$-\nabla^{2} \mathrm{~B}_{\mathrm{x}}=\frac{1}{\gamma}\left[\frac{\partial \gamma}{\partial \mathrm{y}}\left(\frac{\partial \mathrm{B}_{\mathrm{y}}}{\partial \mathrm{x}}-\frac{\partial \mathrm{B}_{\mathrm{x}}}{\partial \mathrm{y}}\right)-\frac{\partial \gamma}{\partial \mathrm{z}}\left(\frac{\partial \mathrm{B}_{\mathrm{x}}}{\partial \mathrm{z}}-\frac{\partial \mathrm{B}_{\mathrm{z}}}{\partial \mathrm{x}}\right)\right]-\mathrm{i} \omega \mu \gamma \mathrm{B}_{\mathrm{x}}$

$$
-\nabla^{2} \mathrm{~B}_{\mathrm{y}}=\frac{1}{\gamma}\left[\frac{\partial \gamma}{\partial \mathrm{z}}\left(\frac{\partial \mathrm{B}_{\mathrm{z}}}{\partial \mathrm{y}}-\frac{\partial \mathrm{B}_{\mathrm{y}}}{\partial \mathrm{z}}\right)-\frac{\partial \gamma}{\partial \mathrm{x}}\left(\frac{\partial \mathrm{B}_{\mathrm{y}}}{\partial \mathrm{x}}-\frac{\partial \mathrm{B}_{\mathrm{x}}}{\partial \mathrm{y}}\right)\right]-\mathrm{i} \omega \mu \gamma \mathrm{B}_{\mathrm{y}}
$$

Multiplying the $y$ component by $i$, and subtracting from the $x$ component gives

$$
\begin{aligned}
-2 \nabla^{2} \mathrm{~B}_{1}^{-}= & \frac{1}{\gamma}\left[\frac{\partial \gamma}{\partial \mathrm{y}}\left(\frac{\partial \mathrm{B}_{\mathrm{y}}}{\partial \mathrm{x}}-\frac{\partial \mathrm{B}_{\mathrm{x}}}{\partial \mathrm{y}}\right)-\frac{\partial \gamma}{\partial \mathrm{z}}\left(2 \frac{\partial \mathrm{B}_{1}^{+}}{\partial \mathrm{z}}-\frac{\partial \mathrm{B}_{\mathrm{z}}}{\partial \mathrm{x}}+\mathrm{i} \frac{\partial \mathrm{B}_{\mathrm{z}}}{\partial \mathrm{y}}\right)\right. \\
& \left.+\frac{\partial \gamma}{\partial \mathrm{x}} \mathrm{i}\left(\frac{\partial \mathrm{B}_{\mathrm{y}}}{\partial \mathrm{x}}-\frac{\partial \mathrm{B}_{\mathrm{x}}}{\partial \mathrm{y}}\right)\right]-\mathrm{i} 2 \omega \mu \gamma \mathrm{B}_{1}^{-}
\end{aligned}
$$

where $B_{1}^{-}=\left(B_{x}-i B_{y}\right) / 2$. Using this $B_{1}^{-}$definition and Gauss's law $\nabla \cdot \mathbf{B}=\mathbf{0}$, we can write one of the terms in Eq. [A3] as

$$
\left(\frac{\partial \mathrm{B}_{\mathrm{y}}}{\partial \mathrm{x}}-\frac{\partial \mathrm{B}_{\mathrm{x}}}{\partial \mathrm{y}}\right)=2 \mathrm{i}\left(\frac{\partial \mathrm{B}_{1}^{-}}{\partial \mathrm{x}}+\mathrm{i} \frac{\partial \mathrm{B}_{1}^{-}}{\partial \mathrm{y}}+\frac{1}{2} \frac{\partial \mathrm{B}_{1}^{-}}{\partial \mathrm{z}}\right)
$$

Substituting Eq. [A4] in Eq. [A3] gives

$$
\begin{aligned}
-\nabla^{2} \mathrm{~B}_{1}^{-}= & \frac{1}{\gamma}\left[\frac{\partial \gamma}{\partial \mathrm{x}}\left(\frac{\partial \mathrm{B}_{1}^{-}}{\partial \mathrm{x}}+\mathrm{i} \frac{\partial \mathrm{B}_{1}^{-}}{\partial \mathrm{y}}+\frac{1}{2} \frac{\partial \mathrm{B}_{\mathrm{z}}}{\partial \mathrm{z}}\right)\right. \\
& +\frac{\partial \gamma}{\partial \mathrm{y}}\left(-\mathrm{i} \frac{\partial \mathrm{B}_{1}^{-}}{\partial \mathrm{x}}+\frac{\partial \mathrm{B}_{1}^{-}}{\partial \mathrm{y}}-\mathrm{i} \frac{1}{2} \frac{\partial \mathrm{B}_{\mathrm{z}}}{\partial \mathrm{z}}\right) \\
& \left.+\frac{\partial \gamma}{\partial \mathrm{z}}\left(-\frac{1}{2} \frac{\partial \mathrm{B}_{\mathrm{z}}}{\partial \mathrm{x}}+\mathrm{i} \frac{1}{2} \frac{\partial \mathrm{B}_{\mathrm{z}}}{\partial \mathrm{y}}+\frac{\partial \mathrm{B}_{1}^{-}}{\partial \mathrm{z}}\right)\right]-\mathrm{i} \omega \mu \gamma \mathrm{B}_{1}^{-}
\end{aligned}
$$

Substituting the partial derivatives of $\gamma$ terms with the derivatives of $\ln (\gamma)$ gives the $B_{1}^{-}$-based cr-MREPT formula in the logarithm form

$$
\boldsymbol{\beta}^{-} \cdot \nabla \ln (\gamma)-\nabla^{2} \mathrm{~B}_{1}^{-}+\mathrm{i} \omega \mu \gamma \mathrm{B}_{1}^{-}=0
$$

where

$$
\begin{gathered}
\gamma=\sigma+\mathrm{i} \omega \epsilon, \quad \mu=\mu_{0}, \quad \nabla \ln (\gamma)=\left[\begin{array}{c}
\frac{\partial \ln (\gamma)}{\partial \mathrm{x}} \\
\frac{\partial \ln (\gamma)}{\partial \mathrm{y}} \\
\frac{\partial \ln (\gamma)}{\partial \mathrm{z}}
\end{array}\right], \\
\boldsymbol{\beta}^{-}=\left[\begin{array}{c}
\beta_{\mathrm{x}}^{-} \\
\beta_{\mathrm{y}}^{-} \\
\beta_{\mathrm{z}}^{-}
\end{array}\right]=\left[\begin{array}{c}
\frac{\partial \mathrm{B}_{1}^{-}}{\partial \mathrm{x}}+\mathrm{i} \frac{\partial \mathrm{B}_{1}^{-}}{\partial \mathrm{y}}+\frac{1}{2} \frac{\partial \mathrm{B}_{\mathrm{z}}}{\partial \mathrm{z}} \\
-\mathrm{i} \frac{\partial \mathrm{B}_{1}^{-}}{\partial \mathrm{x}}+\frac{\partial \mathrm{B}_{1}^{-}}{\partial \mathrm{y}}-\mathrm{i} \frac{1}{2} \frac{\partial \mathrm{B}_{\mathrm{z}}}{\partial \mathrm{z}} \\
-\frac{1}{2} \frac{\partial \mathrm{B}_{\mathrm{z}}}{\partial \mathrm{x}}+\mathrm{i} \frac{1}{2} \frac{\partial \mathrm{B}_{\mathrm{z}}}{\partial \mathrm{y}}+\frac{\partial \mathrm{B}_{1}^{-}}{\partial \mathrm{z}}
\end{array}\right]
\end{gathered}
$$

The $\mathrm{B}_{1}^{+}$-based version can be derived in similar way. Both are given in Eq. [3].

\section{APPENDIX B: DERIVATION OF THE CENTRAL EQUATION}

Writing the imaginary terms in Eq. [13] gives 


$$
\begin{aligned}
& \left(\left[\begin{array}{c}
\frac{\partial}{\partial \mathbf{x}} \phi^{\operatorname{tr}} \\
\frac{\partial}{\partial y} \phi^{\operatorname{tr}} \\
\frac{\partial}{\partial z} \phi^{\operatorname{tr}}
\end{array}\right] \cdot\left[\begin{array}{c}
\frac{\partial \operatorname{Re}\{\ln (\gamma)\}}{\partial \mathbf{x}} \\
\frac{\partial \operatorname{Re}\{\ln (\gamma)\}}{\partial \mathrm{y}} \\
\frac{\partial \operatorname{Re}\{\ln (\gamma)\}}{\partial z}
\end{array}\right]\right) \\
& +\left(\left[\begin{array}{c}
\frac{\partial}{\partial \mathrm{y}}\left(\phi^{+}-\phi^{-}\right) \\
-\frac{\partial}{\partial \mathrm{x}}\left(\phi^{+}-\phi^{-}\right) \\
0
\end{array}\right] \cdot\left[\begin{array}{c}
\frac{\partial \operatorname{Im}\{\ln (\gamma)\}}{\partial \mathrm{x}} \\
\frac{\partial \operatorname{Im}\{\ln (\gamma)\}}{\partial \mathrm{y}} \\
\frac{\partial \operatorname{Im}\{\ln (\gamma)\}}{\partial \mathrm{z}}
\end{array}\right]\right) \\
& -\nabla^{2} \phi^{\text {tr }}+2 \omega \mu_{0} \sigma=0
\end{aligned}
$$

It is observed in the simulations (including the human head simulations) that the derivatives of the phase difference $\left(\phi^{+}-\phi^{-}\right)$are smaller when compared with the derivatives of the transceive phase $\left(\phi^{\mathrm{tr}}\right)$. We also compared the first two terms in Eq. [B1] and found that the term $\left(\left[\begin{array}{c}\frac{\partial}{\partial \mathrm{y}}\left(\varphi^{+}-\varphi^{-}\right) \\ -\frac{\partial}{\partial \mathrm{x}}\left(\varphi^{+}-\varphi^{-}\right) \\ 0\end{array}\right] \cdot\left[\begin{array}{c}\frac{\partial \operatorname{Im}\{\ln (\gamma)\}}{\partial \mathrm{x}} \\ \frac{\partial \operatorname{Im}\{\ln (\gamma)\}}{\partial \mathrm{y}} \\ \frac{\partial \operatorname{Im}\{\ln (\gamma)\}}{\partial \mathrm{z}}\end{array}\right]\right)$ is at least one order of magnitude smaller when compared with the term $\left(\left[\begin{array}{c}\frac{\partial}{\partial \mathrm{x}} \varphi^{\operatorname{tr}} \\ \frac{\partial}{\partial \mathrm{y}} \varphi^{\operatorname{tr}} \\ \frac{\partial}{\partial \mathrm{z}} \varphi^{\operatorname{tr}}\end{array}\right] \cdot\left[\begin{array}{c}\frac{\partial \operatorname{Re}\{\ln (\gamma)\}}{\partial \mathrm{x}} \\ \frac{\partial \operatorname{Re}\{\ln (\gamma)\}}{\partial \mathrm{y}} \\ \frac{\partial \operatorname{Re}\{\ln (\gamma)\}}{\partial \mathrm{z}}\end{array}\right]\right)$. This is true for both transmit-receive configurations that we considered, namely, transmitting from birdcage coil and receiving either from a birdcage coil or from a phased array coil. Neglecting the second term and rewriting Eq. [B1] gives

$$
\left[\begin{array}{l}
\frac{\partial}{\partial \mathrm{x}} \varphi^{\operatorname{tr}} \\
\frac{\partial}{\partial \mathrm{y}} \varphi^{\operatorname{tr}} \\
\frac{\partial}{\partial \mathrm{z}} \varphi^{\operatorname{tr}}
\end{array}\right] \cdot\left[\begin{array}{l}
\frac{\partial \operatorname{Re}\{\ln (\sigma)\}}{\partial \mathrm{x}} \\
\frac{\partial \operatorname{Re}\{\ln (\sigma)\}}{\partial \mathrm{y}} \\
\frac{\partial \operatorname{Re}\{\ln (\sigma)\}}{\partial \mathrm{y}}
\end{array}\right]-\nabla^{2} \varphi^{\mathrm{tr}}+2 \omega \mu_{0} \sigma=0
$$

where

$$
\operatorname{Re}\{\ln (\gamma)\}=\ln \left(\sqrt{\sigma^{2}+(\omega \epsilon)^{2}}\right)
$$

For $\sigma^{2} \gg(\omega \epsilon)^{2}$, the real part of $\ln (\gamma)$ can be approximated as $\operatorname{Re}\{\ln (\gamma)\} \approx \ln (\sigma)$. Defining $\rho=1 / \sigma$ (resistivity), then $\nabla \ln (\sigma)=-\frac{1}{\rho} \nabla \rho$. Substituting these terms in Eq. [B2] and multiplying Eq. [B2] with $-\rho$ yields our central equation

$$
\left(\nabla \varphi^{\mathrm{tr}} \cdot \nabla \rho\right)+\nabla^{2} \varphi^{\mathrm{tr}} \rho-2 \omega \mu_{0}=0
$$

\section{REFERENCES}

1. Chaudhary SS, Mishra RK, Swarup A, Thomas JM. Dielectric properties of normal \& malignant human breast tissues at radiowave \& microwave frequencies. Indian J Biochem Biophys 1984;21:76-79.

2. Surowiec AJ, Stuchly SS, Barr JB, Swarup A. Dielectric properties of breast carcinoma and the surrounding tissues. IEEE Trans Biomed Eng 1988;35:257-263.

3. Joines WT, Zhang Y, Li C, Jirtle RL. The measured electrical properties of normal and malignant human tissues from 50 to $900 \mathrm{MHz}$. Med Phys 1994;21:547-550.

4. Salinas FS, Lancaster JL, Fox PT. 3D modeling of the total electric field induced by transcranial magnetic stimulation using the boundary element method. Phys Med Biol 2009;54:3631-3647.

5. Balidemaj E, Van Lier AL, Crezee H, Nederveen AJ, Stalpers LJA, Van den Berg Cat. Feasibility of electric property tomoghraphy of pelvic tumors at 3T. Magn Reson Med 2015;73:1505-1513.

6. Kwon OI, Chauhan M, Kim HJ, Jeong WC, Wi H, Oh TI, Woo EJ. Fast conductivity imaging in magnetic resonance electrical impedance tomography (MREIT) for RF ablation monitoring. Int J Hyperthermia 2014;30:447-455.

7. Romsauerova A, Ewan M, Horesh L, Oh TI, Woo EJ, Holder DS. A comparison of multi-frequency EIT systems intended for acute stroke imaging. World Congress on Medical Physics and Biomedical Engineering, 2007. p. 3918-3920.

8. Barber DC, Brown BH. Applied potential tomography. J Phys E Sci Instr 1984;17:723-733.

9. Fuks LF, Cheney M, Isaacson D, Gisser DG, Newell JC. Detection and imaging of electric conductivity and permittivity at low frequency. IEEE Trans Biomed Eng 1991;38:1106-1110.

10. Edic PM, Saulnier GJ, Newell JC, Isaacson D. A real-time electrical impedance tomograph. IEEE Trans Biomed Eng 1995;42:849-859.

11. Gençer NG, Ider YZ, Williamson SJ. Electrical impedance tomography: induced-current imaging achieved with a multiple coil system. IEEE Trans Biomed Eng 1996;43:139-149.

12. Griffiths H. Magnetic induction tomography. Meas Sci Technol 2001; 12:1126-1131.

13. Ider YZ, Onart S. Algebraic reconstruction for 3D magnetic resonance-electrical impedance tomography (MREIT) using one component of magnetic flux density. Physiol Meas 2004;25:281-294.

14. Birgul O, Ider YZ. Use of the magnetic field generated by the internal distribution of injected currents for electrical impedance tomography. In Proceedings of 9th Int. Conf. Elec. Bio-Impedance, Heidelberg, Germany, 1995. p 418-419.

15. Ider YZ, Birgul O. Use of the magnetic field generated by the internal distribution of injected currents for electrical impedance tomography (MR-EIT). Elektrik 1998;6:215-225.

16. Seo JK, Kwon O, Woo EJ. Magnetic resonance electrical impedance tomography (MREIT): conductivity and current density imaging. J Phys Conf Ser 2005;12:140-155.

17. Woo EJ, Seo JK. Magnetic resonance electrical impedance tomography (MREIT) for high-resolution conductivity imaging. Physiol Meas 2008;29:R1-R26.

18. Seo JK, Yoon JR, Woo EJ, Kwon O. Reconstruction of conductivity and current density images using only one component of magnetic field measurements. IEEE Trans Biomed Eng 2003;97: 1121-1124.

19. Nam HS, Park C, Kwon OI. Non-iterative conductivity reconstruction algorithm using projected current density in MREIT. Phys Med Biol 2008;53:6947-6961.

20. Scott GC, Joy MLG, Armstrong RL, Henkelman RM. Measurement of nonuniform current density by magnetic resonance. IEEE Trans Med Imaging 1991;10:362-374.

21. Katscher U, Voigt T, Findeklee C, Vernickel P, Nehrke K, Dossel O. Determination of electric conductivity and local SAR via B1 mapping. IEEE Trans Med Imag 2009;28:1365-1374.

22. Haacke EM, Petropoulos LS, Nilges EW, Wu DH. Extraction of conductivity and permittivity using magnetic resonance imaging. Phys Med Biol 1991;36:723-734.

23. Wen H. Noninvasive quantitative mapping of conductivity and dielectric distributions using RF wave propagation effects in highfield MRI. In Proceedings SPIE 5030, Medical Imaging: Physics of Medical Imaging, San Diego, CA, 2003. p. 471-477.

24. Hoult DI. The principle of reciprocity in signal strength calculations-a mathematical guide. Concepts Magn Reson 2000;12:173-187. 
25. Hafalir FS, Oran OF, Gurler N, Ider YZ. Convection-reaction equation based magnetic resonance electrical properties tomography (crMREPT). IEEE Trans Med Imag 2014;33:777-793.

26. Liu J, Zhang X, Schmitter S, Van de Moortele PF, He B. Gradientbased electrical properties tomography (gEPT): a robust method for mapping electrical properties of biological tissues in vivo using magnetic resonance imaging. Magn Reson Med 2015;74:634-646.

27. Balidemaj E, van den Berg C, Trinks J, van Lier AL, Nederveen A, Stalpers L, Crezee H, Remis R. CSI-EPT: a contrast source inversion approach for improved MRI-based electric properties tomography. IEEE Trans Med Imag 2015;34:1788-1796.

28. Cunningham CH, Pauly JM, Nayak KS. Saturated double-angle method for rapid B1 + mapping. Magn Reson Med 2006;55:1326-1333.

29. Voigt T, Nehrke K, Doessel O, Katscher U. T1 corrected B1 mapping using multi-TR gradient echo sequences. Magn Reson Med 2010;64: 725-733.

30. Yarnykh VL. Actual flip-angle imaging in the pulsed steady state: a method for rapid three-dimensional mapping of the transmitted radiofrequency field. Magn Reson Med 2007;57:192-200.

31. Sacolick LI, Wiesinger F, Hancu I, Vogel MW. B1 mapping by BlochSiegert shift. Magn Reson Med 2010;63:1315-1322.

32. Sodickson DK, Alon L, Deniz CM, et al. Local Maxwell tomography using transmit-receive coil arrays for contact-free mapping of tissue electrical properties and determination of absolute RF phase. In Proceedings of the 20th Annual Meeting of ISMRM, Melbourne, Australia, 2012. Abstract 387.

33. Sodickson DK, Alon L, Deniz CM, Ben-Eliezer N, Cloos M, Sodickson LA, Collins CM, Wiggins GC, Novikov DS. Generalized local Maxwell tomography for mapping of electrical property gradients and tensors. In Proceedings of the 21st Annual Meeting of ISMRM, Salt Lake City, Utah, USA, 2013. Abstract 4175.

34. Zhang X, Van de Moortele P-F, Schmitter S, He B. Complex B1 mapping and electrical properties imaging of the human brain using a 16channel transceiver coil at 7T. Magn Reson Med 2013;69:1285-1296.

35. Marques JP, Sodickson DK, Ipek O, Collins CM, Gruetter R. Single acquisition electrical property mapping based on relative coil sensitivities: a proof-of-concept demonstration. Magn Reson Med 2015;74: 185-195.

36. Voigt T, Katscher U, Doessel O. Quantitative conductivity and permittivity imaging of the human brain using electric properties tomography. Magn Reson Med 2011;66:456-466.

37. Katscher U, Kim D-H, Seo JK. Recent progress and future challenges in MR electric properties tomography. Comput Math Methods Med 2013;2013:546-562.

38. Van Lier AL, Brunner DO, Pruessmann KP, Klomp DWJ, Luijten PR, Lagendijk JJW, van den Berg CAT. B1+Phase mapping at $7 \mathrm{~T}$ and its application for in vivo electrical conductivity mapping. Magn Reson Med 2012;67:552-561.

39. Lee SK, Bulumulla S, Hancu I. Theoretical investigation of random noiselimited signal-to-noise ratio in MR-based electrical properties tomography. IEEE Trans Med Imag 2015;34:2220-2232.

40. Gurler N, Oran OF, Ider YZ. cr-MREPT using multichannel receive coil. In Proceedings of the 22nd Annual Meeting of ISMRM, Milan, Italy, 2014. Abstract 3247 .
41. John V, Knobloch P. On spurious oscillations at layers diminishing (SOL D) methods for convection-diffusion equations. Part I: a review. Comput Method Appl M 2007;196:2197-2215.

42. John V, Knobloch P. On spurious oscillations at layers diminishing (SOL D) methods for convection-diffusion equations. Part II: analysis for P1 and Q1 finite elements. Comput Method Appl M 2008;197: 1997-2014.

43. Gurler N, Ider YZ. Numerical methods and software tools for simulation, design, and resonant mode analysis of radio frequency birdcage coils used in MRI. Concepts Magn Reson Part B 2015;45:13-32.

44. Gudbjartsson H, Patz S. The Rician distribution of noisy MRI data. Magn Reson Med 1995;34:910-914 [erratum Magn Reson Med 1996; 36:332].

45. Scott GC, Joy MLG, Armstrong RL, Henkelman M. Sensitivity of magnetic resonance current-density imaging. J Magn Reson 1992;97:235254.

46. Stogryn A. Equations for calculating the dielectric constant of saline water. IEEE Trans Microwave Theory Tech 1971;19:733-736.

47. Iizuka K. An agar-agar chamber for the study of electromagnetic waves in an homogenous medium. IEEE Trans Antennas Propag 1971;19:365-377.

48. Katscher U, Abe H, Ivancevic MK, Djamshidi K, Karkowski P, Newstead G. Towards the investigation of breast tumor malignancy via electric conductivity measurement. In Proceedings of the 21st Annual Meeting of ISMRM, Salt Lake City, Utah, USA, 2013. Abstract 3372 .

49. Dietrich O, Raya JG, Reeder SB, Reiser MF, Schoenberg SO. Measurement of signal-to-noise ratios in MR images: influence of multichannel coils, parallel imaging, and reconstruction filters. J Magn Reson Imaging 2007;26:375-385.

50. Bangerter NK, Hargreaves BA, Vasanawala SS, Pauly JM, Gold GE, Nishimura DG. Analysis of multiple-acquisition SSFP. Magn Reson Med 2004;51:1038-1047.

51. Gabriel S, Lau RW, Gabriel C. The dielectric properties of biological tissues. Part III: Parametric models for the dielectric spectrum of tissues. Phys Med Biol 1996;41:2271-2293.

52. Katscher U, van Lier AL, van den Berg C, Keupp J. RF shimming improves phase-based conductivity imaging. In Proceedings of the 20th Annual Meeting of ISMRM, Melbourne, Australia, 2012. Abstract 3487.

53. Katscher U, Braun M, Findeklee C, Leussler C, Graesslin I, Vernickel P, Morlock M. B1-based SAR determination for local RF transmit coils. In Proceedings of the 23rd Annual Meeting of ISMRM, Toronto, Canada, 2015. Abstract 383.

54. Oran OF, Ider YZ. Magnetic resonance electrical impedance tomography (MREIT) based on the solution of the convection equation using FEM with stabilization. Phys Med Biol 2012;57:5113-5140.

55. Minhas AS, Kim YT, Kim HJ, Woo EJ, Kim M, Kim DH, Seo JK. Feasibility of dual-frequency conductivity imaging using MREIT and MREPT. In Proceedings of the NFSI \& ICBEM, Banff, Canada, 2011. p. 68-71.

56. Kim HJ, Jeong WC, Sajib SZK, Kim MO, Kwon OI, Woo EJ, Kim DH. Simultaneous imaging of dual-frequency electrical conductivity using a combination of MREIT and MREPT. Magn Reson Med 2014;71:200208. 